\title{
Linear stability analysis of a horizontal phase boundary separating two miscible liquids
}

\author{
Abdesselem Kheniene and Anatoliy Vorobev* \\ University of Southampton, Faculty of Engineering and the Environment, Southampton SO17 1BJ, UK
}

(Received 25 June 2013; published 19 August 2013)

\begin{abstract}
The evolution of small disturbances to a horizontal interface separating two miscible liquids is examined. The aim is to investigate how the interfacial mass transfer affects development of the Rayleigh-Taylor instability and propagation and damping of the gravity-capillary waves. The phase-field approach is employed to model the evolution of a miscible multiphase system. Within this approach, the interface is represented as a transitional layer of small but nonzero thickness. The thermodynamics is defined by the Landau free energy function. Initially, the liquid-liquid binary system is assumed to be out of its thermodynamic equilibrium, and hence, the system undergoes a slow transition to its thermodynamic equilibrium. The linear stability of such a slowly diffusing interface with respect to normal hydro- and thermodynamic perturbations is numerically studied. As a result, we show that the eigenvalue spectra for a sharp immiscible interface can be successfully reproduced for long-wave disturbances, with wavelengths exceeding the interface thickness. We also find that thin interfaces are thermodynamically stable, while thicker interfaces, with the thicknesses exceeding an equilibrium value, are thermodynamically unstable. The thermodynamic instability can make the configuration with a heavier liquid lying underneath unstable. We also find that the interfacial mass transfer introduces additional dissipation, reducing the growth rate of the Rayleigh-Taylor instability and increasing the dissipation of the gravity waves. Moreover, mutual action of diffusive and viscous effects completely suppresses development of the modes with shorter wavelengths.
\end{abstract}

DOI: 10.1103/PhysRevE.88.022404

PACS number(s): 68.05.-n, 47.35.-i, 64.70.Ja, 52.35.Py

\section{INTRODUCTION}

We consider an evolution of small disturbances to a horizontal flat interface separating two liquids of different densities and viscosities. It is well known that for an immiscible interface such a configuration is unstable and subject to the Rayleigh-Taylor instability if a lower liquid is lighter. In the opposite case of a heavier lower liquid, the configuration is hydrodynamically stable and the small disturbances generate gravity-capillary waves.

The stability of immiscible interfaces was first studied by Rayleigh [1] who developed the linear stability theory for a plane interface and derived the dispersion relation for normal disturbances. This theoretical work was continued by a number of researchers who examined viscosity and capillarity effects on development of small perturbations [2-6], and the results were experimentally confirmed in, e.g., [7-9]. The current focus of the research community is shifted into investigation of a nonlinear evolution of the Rayleigh-Taylor instability and dynamics of waves of larger amplitudes [10,11].

In our work we examine the evolution of small disturbances applied to an interface separating two miscible liquids. The liquids represent the components of a binary mixture. We assume that initially a binary mixture is out its thermodynamic equilibrium, and its equilibration is driven by the diffusion process and hydrodynamic flows. Development of the hydrodynamic modes usually occurs considerably faster as the diffusive time scales are typically considerably larger than the hydrodynamic time scales. This normally allows treating the interface between the slowly miscible liquids as an immiscible boundary. In the current work, we however aim to investigate the role exerted by the interfacial diffusion on the

*A.Vorobev@soton.ac.uk stability of a phase boundary, and hence we would assume that the diffusive and hydrodynamic time scales are comparable. This in particular is true for the binary mixtures with stronger diffusion, or for the systems with suppressed hydrodynamic modes (e.g., very viscous liquids or the interfaces trapped in a microcavity).

Technological applications involving diffusive and hydrodynamic processes in multiphase systems undergoing phase transitions are ubiquitous. These include chemical vegetable extraction [12], soil remediation [13,14], and enhanced oil recovery (miscible displacement of oil from porous media [15]), which can be roughly reduced to the same physical process: The porous volume is initially saturated by a liquid solute that needs to be dissolved or displaced. It is known that miscible liquids in confined geometries (within pores) are difficult to mix up, and mixing (molecular co-exchange between solute and solvent) is accelerated by hydrodynamic instabilities. Hence, efficiencies of the whole processes are determined by shapes of solute-solvent interfaces, rates of interfacial mass transport, hydrodynamic flows near the interface, etc. The similar fluid physics also defines a variety of other phenomena, such as mixing of fresh and salt water or mixing of chemical spills into sea water.

Despite the fundamental importance of the liquid-liquid diffusion and of its seeming simplicity, its understanding is still missing $[16,17]$. It is known that diffusion in liquid-liquid binary mixtures differs from diffusion in gaseous mixtures (where intermolecular forces are negligible), or from diffusion of a minor impurity dissolved in a liquid solvent. The diffusion in these cases is well defined by the classical Fick's law, with the diffusion flux being proportional to the concentration gradient. The Fick's law however does not properly define the diffusion of liquid-liquid interfaces. Complexities of the liquid-liquid interface diffusion stem from the difference in intermolecular forces in the continua of the neighboring 
liquids leading to the capillary effects which define both the interface morphology and the interfacial mass transfer (as for the interfacial diffusion to occur, molecules need to possess sufficient kinetic energy in order to overcome the interfacial potential barrier created by the intermolecular forces). We may say that, in immiscible liquids, the interfacial diffusion is absent due to the strong capillary effects, while in miscible systems the values of the surface tension coefficient are considerably lower signifying the lower potential barriers. An example is honey immersed in a cup of tea: Honey and water are completely miscible, but honey remains clearly visible for a long period of time, with the mass exchange between liquids occurring through the liquid-liquid interface. Being however agitated a honey-droplet interface keeps its shape indicating that the miscible interface is endowed with the surface tension. The concept of the dynamic surface tension between miscible liquids was first discussed and later experimentally confirmed in, e.g., Refs. [18-23].

It is generally accepted that the diffusion flux through the liquid-liquid interface is to be defined by the generalized Fick's law, i.e., through the gradient of the chemical potential. An equilibrium binary mixture is defined by the equal chemical potentials in neighboring phases, and there is no mass flux across the interface despite existence of the strong concentration gradient across the phase boundary. By taking a binary mixture out of its equilibrium (e.g., by a temperature quench), one induces the interfacial mass flux, which intensity is determined by the gradient of the chemical potential. Such a description includes, in particular, the effects of barodiffusion $[24,25]$. The ways how the diffusive flux is defined is a key difference of the current paper from the other studies, which were also focused on the stability of miscible interfaces but defined the diffusion flux through the classical Fick's law (for instance, [26-28]).

Currently, there are several different approaches that can be employed for tracking an evolving multiphase system. The classical Laplace approach (also called the sharp-interface approach) represents a phase boundary as an infinitely thin surface of discontinuity endowed with some macroscopic properties (such as surface tension). Two sets of equations are separately solved within each phase and solutions are matched by using the proper boundary conditions $[6,29]$. This approach is well grounded as typically the thickness of a phase boundary is just several molecular layers, i.e., zero for the macroscopic hydrodynamic theory. On the basis of this approach the initial development of the Rayleigh-Taylor instability and small-amplitude gravity-capillary waves at an interface between two immiscible liquids were successfully described [2-6,29]. The Laplace approach is however inconvenient and, moreover, not feasible for the problems where the interface shape experiences strong (e.g., waves of larger amplitude) or topological changes (such as nonlinear development of the Rayleigh-Taylor instability, breakup and coalescence of droplets, etc.). To tackle similar problems new computational approaches were developed; these are the volume-of-fluid [30], level-set [31], and phase-field methods [32-36]. The core idea of these numerical approaches is that the entire multiphase system (all phases and the phase boundary) is represented as a continuous medium, and its evolution is defined by one set of governing equations. The volume-of-fluid and the level-set methods however introduce new scalar functions, which are used to trace the interface position, on the basis of the mathematical grounds. The phase-field method instead uses a natural variable (e.g., concentration or density) for the same purposes, so the governing equations are physics based and in particular include the surface tension both in the momentum and diffusion equations [24,25]. In the current work, we use the phase-field approach to investigate the linear stability of a miscible interface.

The phase-field method was already used to model the Rayleigh-Taylor instability, and the gravity-capillary waves at an immiscible interface [33-37]. For instance, the authors of Refs. [35,37] proved through direct numerical simulations that the classical observations, which were previously obtained on the basis of the Laplace approach, could be successfully reproduced. In [38], the classical dispersion relations for the Rayleigh-Taylor instability were rederived from the phasefield governing equations for immiscible interfaces in the limit of vanishing interface thickness.

In [39-41], the thermodynamic stability of an equilibrium interface (also called a kink solution) was studied. It was found that such an interface is stable in respect to normal perturbations with the decay rate growing as $k^{3}$. The linear stability of the diffusive interfaces was examined in [42], however, for the interfaces located within porous medium and defined by the reduced equations for the porous medium. It was shown that the diffusivity's role is reduced to additional dissipation. In the current work, we study the linear stability of a horizontal phase boundary between two miscible liquids.

The present paper is organized as follows. The general mathematical model for a miscible multiphase system is defined in Sec. II. In the same section, we also define a basic state and introduce small amplitude normal perturbations for which the set of linearized amplitude equations is derived. The full equations are solved in three stages. In Sec. III, the diffusive terms are excluded, assuming that the Peclet number is infinitely high, and the purely hydrodynamic perturbations are examined. In the absence of diffusion the interface is immiscible, but it is still represented as a layer of the finite thickness. Attention is given to the limit of vanishing interface thickness, in order to obtain the restrictions on the value of the interface thickness which allow an accurate reproduction of the sharp-interface eigenvalue spectrum. In Sec. IV, we consider a system with the suppressed hydrodynamic perturbations, with the evolution solely defined by the diffusion equation. The aim is to understand the stability of the interface itself towards purely thermodynamic perturbations. Section V combines these studies by considering the stability of miscible interfaces. Finally, Sec. VI presents the summary and discussion of the obtained results.

\section{MATHEMATICAL MODEL}

\section{A. Governing equations}

We study the evolution of a binary mixture, with components being called liquid 1 and liquid 2 . The concentration field $C$ is introduced as a mass fraction of liquid 2 in a mixture. Mixture density is a function of concentration, and the simplest relation between density and concentration can be written for 
the so-called simple mixtures,

$$
\frac{1}{\rho}=\frac{1-C}{\rho_{1}}+\frac{C}{\rho_{2}} .
$$

Here $\rho$ is the mixture density, while $\rho_{1}$ and $\rho_{2}$ are the densities of pure liquids that constitute a mixture. This relation is based on the assumption that changes in volume upon mixing are negligible, which is a good approximation for many mixtures [20]. We use an even simpler, linearized relation that is however valid for almost all liquid-liquid binary mixtures due to a small density contrast typical for such systems,

$$
\rho=\rho_{1}(1-\phi C), \quad \phi \equiv \frac{\rho_{1}-\rho_{2}}{\rho_{2}} .
$$

When pure components of a binary mixture are brought into contact, molecules of the neighboring phases interdiffuse. In thermodynamic equilibrium, achievable after usually a very long time period, the mixture can become homogeneous with the equilibrium concentration defined by the mass balance, or stay heterogeneous with the equilibrium concentrations $C_{1}>0$ and $C_{2}<1$, defined by the phase diagram (at the initial contact, $C_{1}=0$ and $C_{2}=1$ ). The phase diagrams can be very different [43], but the primary focus of the current work is on the binary mixtures with the upper critical solution temperature (e.g., the mixture of isobotyric acid and water $[16,22])$. If the mixture temperature is below the critical value, then the equilibrium state can be heterogeneous or homogeneous depending on the average concentration. If the temperature is above the critical point, then the mixture is always homogeneous in equilibrium. There are also binary mixtures fully miscible under all thermodynamic conditions, such as glycerol-water or soybean oil-hexane. We presume that the evolution of the latter mixtures is similar to the evolution of the mixture with the upper critical point at the above-critical temperatures, and hence, the theory and results of this work are also applicable to such mixtures miscible at all conditions.

The evolution of a binary mixture is defined within the framework of the phase-field approach, initiated by van der Waals [44], Korteweg [45], and Cahn and Hilliard [46], who first proposed to model the whole multiphase system as a continuous medium and to define the free energy as a function of concentration and concentration gradient (for an isothermal system),

$$
f=f_{0}(C)+\frac{\epsilon}{2}(\vec{\nabla} C)^{2} .
$$

Here $f_{0}$ is the classical part of the free energy, and the second term takes into account the capillary effects. The amplitude of the second term is defined by the so-called capillary parameter $\epsilon$, which is so small that the second term can be neglected everywhere except the places of large concentration gradients that define the position of a phase boundary.

The classical part of the free energy function should define the possible states of a binary mixture. In the current work we are interested in the evolution of binary mixtures undergoing phase transformations: Initially, a mixture is heterogeneous, but ultimately could become homogeneous. To define $f_{0}$, we found convenient the classical Landau expression,

$$
f_{0}=a\left(C-C_{c}\right)^{2}+b\left(C-C_{c}\right)^{4},
$$

which was initially proposed as an approximation for a near critical system. We however use this model to describe the thermodynamic evolution of systems both near and far from the critical point. That is, we treat expression (4) as a model with two phenomenological parameters $a$ and $b$, capable of defining the binary system with both homogeneous and heterogeneous equilibrium states. It can be shown that in the vicinity of the critical point parameter $a$ is proportional to $\left(T-T_{c}\right)$, i.e., negative for the temperatures below the critical point and positive for the above-critical temperatures. Parameter $b$ is always positive.

For further discussion, it will be convenient to change the reference point for the concentration field as $\left(C-C_{c}\right) \rightarrow C$. This new definition of concentration will be used in the rest of the paper.

The full set of the Cahn-Hilliard-Navier-Stokes equations that describe the thermo- and hydrodynamic evolution of binary mixtures of two incompressible liquids was first derived by Lowengrub and Truskinovsky [24]. One of the main features of these equations is the quasicompressibility: The full continuity equation should be used to define the evolution of two incompressible liquids due to dependence of the mixture density on concentration. This feature makes the numerical solution of the full equations hardly feasible. It was later shown that the full Cahn-Hilliard-Navier-Stokes equations could be simplified on the basis of the multiple-scale method by separating fast quasiacoustic processes and slow diffusive and convective processes. So, it was shown that the slow evolution of binary mixtures is defined by the Boussinesq approximation [25], which is adopted for the current work.

The governing equations include the balances of mass, momentum, and species, [25]

$$
\begin{aligned}
\vec{\nabla} \cdot \vec{u} & =0, \\
\rho_{1}\left(\frac{\partial \vec{u}}{\partial t}+(\vec{u} \cdot \vec{\nabla}) \vec{u}\right) & =-\vec{\nabla} p+\eta \nabla^{2} \vec{u}-\epsilon \nabla^{2} C \vec{\nabla} C-\phi C \vec{g},
\end{aligned}
$$

$\rho_{1}\left(\frac{\partial C}{\partial t}+(\vec{u} \cdot \vec{\nabla}) C\right)=\alpha \nabla^{2} \mu$.

Here $p$ is the modified pressure field that needs to be determined from an incompressibility constraint, $\vec{u}$ is the velocity, $\alpha$ and $\eta$ are the mobility and viscosity coefficients, and $\vec{g}$ is the gravity acceleration.

One sees that the equations written in the Boussinesq approximation are fully incompressible. The momentum equation includes the Korteweg force that models the effect of the surface tension on the interface morphology. The diffusive mass transport is defined by the general Fick's law, i.e., through the gradient of the chemical potential, which is defined as

$$
\mu=\frac{d f_{0}}{d C}+\phi(\vec{g} \cdot \vec{r})-\epsilon \nabla^{2} C,
$$

where $d f_{0} / d C$ stands for the classical part of the chemical potential. The full expression for the chemical potential indicates that the diffusive mass transport takes into account the barodiffusion effects, i.e., diffusive flux induced by the pressure gradients induced by the gravity and capillary forces.

The governing equations need to be supplemented with the boundary conditions. We consider the interface separating two 
semi-infinite liquid domains, and hence, the perturbations to all variables are assumed to vanish at large enough distances from the phase boundary, which will be used for the boundary conditions.

Next, we nondimensionalize the governing equations by choosing the following scales for time, velocity, pressure, and specific free energy:

$$
\tau_{*}=\frac{L_{*}}{V_{*}}, \quad V_{*}=\mu_{*}^{1 / 2}, \quad p_{*}=\rho_{*} \mu_{*}, \quad f_{*}=\mu_{*} .
$$

Here $L_{*}$ stands for the typical length scale. For the typical density $\rho_{*}$ and chemical potential $\mu_{*}$, we can accept the following values, $\rho_{*}=\rho_{1}$ and $\mu_{*}=b$. In nondimensional form the governing equations then read

$$
\begin{aligned}
& \vec{\nabla} \cdot \vec{u}=0, \\
& \frac{\partial \vec{u}}{\partial t}+(\vec{u} \cdot \vec{\nabla}) \vec{u}=-\vec{\nabla} P+\frac{1}{\operatorname{Re}} \nabla^{2} \vec{u}-\mathrm{Ca}^{2} C \vec{\nabla} C+\mathrm{Gr} C \vec{\gamma} \\
& \frac{\partial C}{\partial t}+(\vec{u} \cdot \vec{\nabla}) C=\frac{1}{\operatorname{Pe}} \nabla^{2} \mu \\
& \mu=-\operatorname{Gr}(\vec{\gamma} \cdot \vec{r})+2 A C+4 C^{3}-\mathrm{Ca}^{2} C
\end{aligned}
$$

Here $\vec{\gamma}$ is a unit vector directed upwards.

The nondimensional parameters entering the above equations are the Peclet number,

$$
\mathrm{Pe}=\frac{\rho_{*} L_{*}}{\alpha \mu_{*}^{1 / 2}} ;
$$

the capillary number, [47]

$$
\mathrm{Ca}=\frac{\epsilon}{\mu_{*} L_{*}^{2}}
$$

the Reynolds number,

$$
\operatorname{Re}=\frac{\rho_{*} \mu_{*}^{1 / 2} L_{*}}{\eta_{*}} ;
$$

and the Grashof number,

$$
\mathrm{Gr}=\phi \frac{g L_{*}}{\mu_{*}} .
$$

Parameter

$$
A=\frac{a}{\mu_{*}}
$$

defines the equilibrium states of the binary system. If $A$ is negative, the system can be homogeneous or heterogeneous in equilibrium; for positive $A$, an equilibrium state of a binary mixture is always homogeneous.

The viscosities of mixture components are different and the mixture viscosity is in general a function of concentration. We however assume that this difference in viscosities is small, so it does not affect the evolution of a mixture. In the definition of the Reynolds number, $\eta_{*}$ can be the viscosity of one of the pure components, e.g., $\eta_{1}$.

\section{B. Basic state. Linear stability analysis}

We study the linear stability of an interface separating two semi-infinite domains occupied by the components of a binary

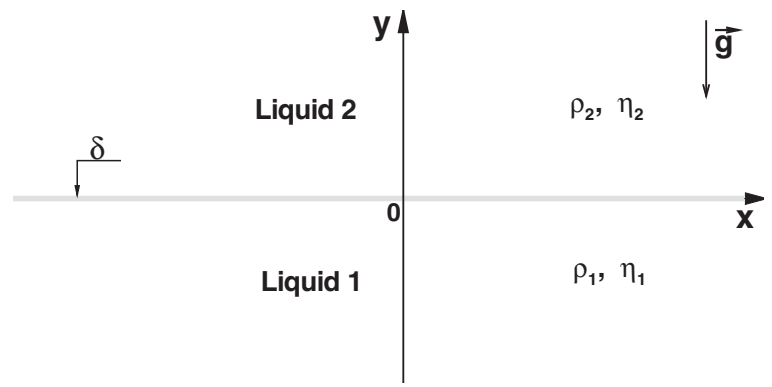

FIG. 1. Two semi-infinite domains of two incompressible miscible liquids of densities $\rho_{1}$ and $\rho_{2}$ and viscosities $\eta_{1}$ and $\eta_{2}$ are separated by an interface of thickness $\delta$.

mixture; see Fig. 1. At an initial contact of two pure mixture components, the concentration profile can be approximated as

$$
C_{0}(y)=\frac{1}{2} \tanh \left(\frac{y}{\delta}\right),
$$

where $\delta$ is the thickness of the interface. The pure mixture components are defined by the values $\pm 1 / 2$. We assume that there is no hydrodynamic motion at this state; i.e., $\vec{u}=0$.

This state does not correspond to a thermodynamic equilibrium of the mixture, and hence the chemical potential is not constant across the layer,

$$
\mu_{0}(y)=\mathrm{Gr} y+2 A C_{0}+4 C_{0}^{3}-\mathrm{Ca} \frac{d^{2} C_{0}}{d y^{2}} .
$$

There is one specific profile, sometimes called a kink solution, which corresponds to a heterogeneous equilibrium state of a binary mixture achievable for negative values of parameter $A$ (and if the gravity effect is neglected). For a kink solution, the equilibrium concentrations of the mixture components are $C_{1,2}= \pm \sqrt{-A / 2}$ and the interface thickness is $\delta_{0} \equiv \sqrt{-\mathrm{Ca} / A}$. In the current paper, the evolution of an initially off-equilibrium binary system is examined, and hence the interface thickness and the capillary parameter are treated as two independent quantities.

We can also introduce the surface tension of the interface as $[24,44,46]$

$$
\sigma=\mathrm{Ca} \int_{-\infty}^{\infty}\left(\frac{d C_{0}}{d y}\right)^{2} d y,
$$

where $y$ is the transversal coordinate. For the introduced basic profile (19), the surface tension is equal to

$$
\sigma=\frac{\mathrm{Ca}}{3 \delta} \text {. }
$$

The response of the basic state to $2 \mathrm{D}$ normal disturbances is studied. To solve Eqs. (10)-(13), the stream function, $\psi$, is introduced that is defined as $\vec{u}=(\partial \psi / \partial y,-\partial \psi / \partial x)$. The stability of a quiescent state with the distributions of the concentration and chemical potential defined by expressions (19) and (20) is examined with respect to normal perturbations,

$$
\begin{aligned}
& \psi(x, y, t) \rightarrow \psi(y) \exp (i k x-i \omega t), \\
& C(x, y, t) \rightarrow C_{0}(y)+C(y) \exp (i k x-i \omega t), \\
& \mu(x, y, t) \rightarrow \mu_{0}(y)+\mu(y) \exp (i k x-i \omega t) .
\end{aligned}
$$


Hereafter, $\psi(y), C(y)$, and $\mu(y)$ denote the amplitudes of perturbations of the stream function, concentration, and chemical potential, respectively. A disturbance is defined by the wave number $k$ ( $k \equiv 2 \pi / \lambda$ with $\lambda$ being the wavelength) and the complex frequency $\omega$ with $\omega_{r}$ being the time frequency ( $\omega_{r} / k$ is the phase speed of waves) and $\omega_{i}$ being the growth or decay rate.

Substitution of these expressions into the governing equations and then retention of the linear terms yields the system of the linearized amplitude equations,

$$
\begin{aligned}
-i \omega\left(\psi^{\prime \prime}-k^{2} \psi\right)= & \frac{1}{\operatorname{Re}}\left(\psi^{i v}-2 k^{2} \psi^{\prime \prime}+k^{4} \psi\right)+i k \mathrm{Ca} \\
& \times\left[\left(C^{\prime \prime}-k^{2} C\right) C_{0}^{\prime}-C_{0}^{\prime \prime \prime} C\right]+i k \mathrm{Gr} C \\
-i \omega C-i k C_{0}^{\prime} \psi= & \frac{1}{\mathrm{Pe}}\left(\mu^{\prime \prime}-k^{2} \mu\right), \\
\mu= & \left(2 A+12 C_{0}^{2}\right) C-\mathrm{Ca}\left(C^{\prime \prime}-k^{2} C\right) .
\end{aligned}
$$

Here a prime denotes differentiation in respect to $y$.

Since an interface separates two semi-infinite domains, all perturbations are to vanish as $y \rightarrow \pm \infty$, which is used as the boundary conditions for the solutions of the amplitude equations. For numerical integration, the depths of the liquid domains were however restricted by two boundaries placed at $\pm L$, with $L$ being sufficiently large to exclude any boundary effects.

We need to mention that the basic profile slowly diffuses, with the interface thickness evolving in time as $\delta \sim$ $\sqrt{t / \mathrm{Pe}}$ to its equilibrium value $\delta_{0}$ if $A<0$ or to infinity if $A \geqslant 0$. This time dependence is however much slower compared to the exponential growth of the introduced normal perturbations. Therefore, we study the time evolution of perturbations on the background of the "frozen" basic state.

The set of the amplitude equations and boundary conditions defines a boundary-value problem. Its numerical solution is not straightforward due to stiffness caused by extreme (too large or too small) values of the governing nondimensional parameters (large Reynolds and Peclet numbers, and small capillary number). We used two numerical methods to solve the problem, the shooting method supplemented with the Gram-Schmidt orthogonalization [48-51] and the compound matrix method [52-54]. The fourth-order adaptive Runge-Kutta technique was used for numerical integration and the Muller's method was employed for root finding.

\section{AN IMMISCIBLE INTERFACE OF FINITE THICKNESS SUBJECT TO HYDRODYNAMIC PERTURBATIONS}

The considered problem involves the interaction of several physical effects, namely the viscosity, diffusivity, and capillarity. We obtain the solution in three main steps and start from consideration of an interface separating two immiscible liquids, i.e., by taking the Peclet number being infinite. For immiscible liquids the amplitude equations (26)-(28) are reduced to one equation of the fourth order,

$$
\begin{aligned}
-i \omega\left(\psi^{\prime \prime}-k^{2} \psi\right)= & \frac{1}{\operatorname{Re}}\left(\psi^{i v}-2 k^{2} \psi^{\prime \prime}+k^{4} \psi\right)-i \frac{k^{2}}{\omega} \mathrm{Ca} C_{0}^{\prime} \\
& \times\left[2 C_{0}^{\prime \prime} \psi^{\prime}-C_{0}^{\prime}\left(\psi^{\prime \prime}-k^{2} \psi\right)\right] \\
& -i \frac{k^{2}}{\omega} \mathrm{Gr} C_{0}^{\prime} \psi .
\end{aligned}
$$

The numerical integration of Eq. (29) was initiated simultaneously at the left and right ends by using the following boundary conditions,

$$
\begin{aligned}
& y=-L: \quad \psi=A_{1} \exp (-k L)+A_{3} \exp (-q L), \\
& y=L: \quad \psi=A_{2} \exp (-k L)+A_{4} \exp (-q L),
\end{aligned}
$$

with

$$
q \equiv \sqrt{k^{2}-i \omega \operatorname{Re}}
$$

Formulas (30) represent the general solutions of Eq. (29), that is simplified under assumption that $C_{0}= \pm 1 / 2$ and $C_{0}^{\prime}=$ $C_{0}^{\prime \prime}=0$, which is true in the limit of $y \rightarrow \pm \infty$. For particular values of the wave number $k$ and parameters $\mathrm{Re}, \mathrm{Gr}$, and $\mathrm{Ca}$, two integration runs for different values of the constants, $A_{1}$, $A_{2}, A_{3}$, and $A_{4}$ were initiated from each boundary and were carried out towards the middle of the layer. At the middle point, the condition of the linear independency of the four obtained solutions generated an eigenvalue $\omega$.

The numerical results were compared against the well known analytical dispersion relations derived on the basis of the Laplacian approach $[6,29]$. For the inviscid case, this is the explicit relation, that has the following form in terms of the parameters of the phase-field approach,

$$
\omega^{2}=\frac{1}{2} \mathrm{Gr} k+\frac{\sigma}{2} k^{3}=\frac{1}{2} \mathrm{Gr} k+\frac{\mathrm{Ca}}{6 \delta} k^{3} .
$$

If viscous effects are taken into account, then the dispersion relation is defined by the implicit relation,

$$
-\frac{q(q+k)\left(q^{2}-k^{2}\right)}{\mathrm{Re}^{2}}=\frac{1}{2} \mathrm{Gr} k+\frac{\mathrm{Ca}}{6 \delta} k^{3},
$$

with $q$ defined by Eq. (32).

One expression [either (33) or (34)] defines the dispersion relations for both the Rayleigh-Taylor instability and the gravity-capillary waves, which differ by the sign of the Grashof number. For $\mathrm{Gr}>0$, a heavier liquid underlies a lighter one, $\rho_{1}>\rho_{2}$, which corresponds to a configuration of the gravity-capillary waves. In this case $\omega$ is complex, with its real part defining the frequency of oscillations and imaginary part defining the damping rate. In the opposite case, $\mathrm{Gr}<0$, the interface is subject to the Rayleigh-Taylor instability, and $\omega$ is always purely imaginary indicating monotonic growth of perturbations. For both cases, the viscous force introduces the dissipation that mostly affects the development of the modes with shorter wavelengths, as well as the capillarity that also predominantly dampens the modes with shorter wavelengths. In the case of the Rayleigh-Taylor instability the short-wavelength modes become stabilized, while in the case of the gravity-capillary waves the short-wavelength modes start to decay strongly and monotonically, i.e., lose their wavelike character. 
(a) RTI

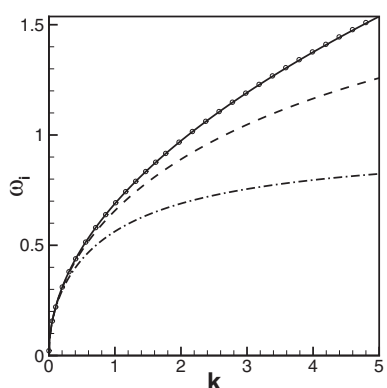

(c) RTI

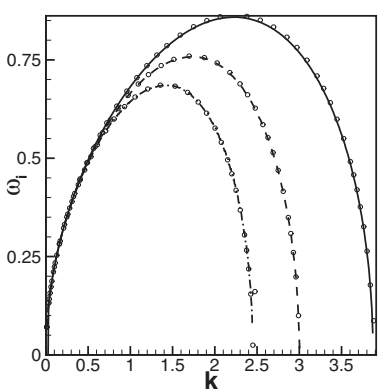

(e) RTI

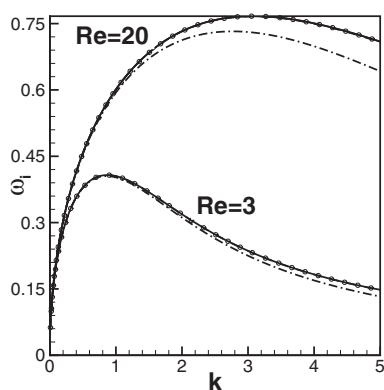

(b) RTI

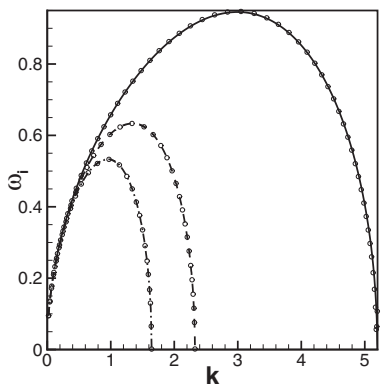

(d) RTI

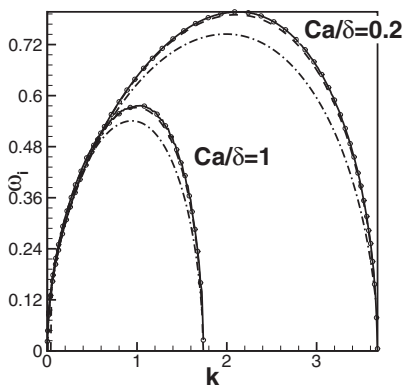

(f) RTI

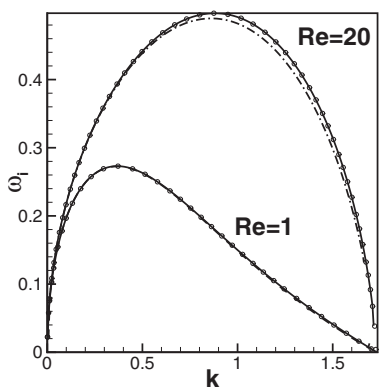

FIG. 2. Rayleigh-Taylor instability at an immiscible interface. The growth rate $\omega_{i}$ versus the wave number $k$ is shown for $\mathrm{Gr}=-1$, and (a) $\mathrm{Ca}=0$ and various interface thicknesses $(\delta=0.5$, dash-dot line; $\delta=0.1$, dashed line; $\delta=0.001$, solid line); (b) $\delta=0.001$ and various capillary numbers $(\mathrm{Ca}=0.001$, dash-dot line; $\mathrm{Ca}=0.0005$, dashed line; $\mathrm{Ca}=0.0001$, solid line); (c) $\mathrm{Ca}=0.005$ and various $\delta$ ( $\delta=0.001$, dash-dot line; $\delta=0.0015$, dashed line; $\delta=0.0025$, solid line); various $\mathrm{Ca}$ and $\delta$ under constant ratio $\mathrm{Ca} / \delta=0.2\left(\mathrm{Ca}=10^{-4}\right.$ and $\delta=5 \times 10^{-4}$, solid line; $\mathrm{Ca}=0.001$ and $\delta=0.005$, dashed line; and $\mathrm{Ca}=0.01$ and $\delta=0.05$, dash-dot line $)$ and $\mathrm{Ca} / \delta=1(\mathrm{Ca}=\delta=$ 0.001 , solid line; $\mathrm{Ca}=\delta=0.01$, dashed line, $\mathrm{Ca}=\delta=0.1$, dash-dot line); (e) $\mathrm{Re}=3$ and $\mathrm{Re}=20, \mathrm{Ca}=0$ and different $\delta$ ( $\delta=0.1$, dash-dot line; $\delta=0.01$, dashed line; $\delta=0.001$, solid line); and (f) $\mathrm{Re}=1$ and $\mathrm{Re}=20$ and different $\mathrm{Ca}$ and $\delta$ under constant ratio $\mathrm{Ca} / \delta=1(\mathrm{Ca}=\delta=0.1$, dash-dot line; $\mathrm{Ca}=\delta=0.01$, dashed line; $\mathrm{Ca}=\delta=0.001$, solid line). (a)-(d) depict inviscid results. Symbol "o" marks the sharp-interface results.

The numerical data for the Rayleigh-Taylor instability, obtained on the basis of the phase-field approach, are plotted in Fig. 2 for the interfaces separating two inviscid [Figs. 2(a)2(d)] and two viscous liquids [Figs. 2(e) and 2(f)]. The results are shown against the curves obtained from the classical formulas (33) and (34). All classical observations for the RayleighTaylor instability can be reproduced. In particular, we observe that the amplitudes of all modes grow monotonically. The

growth rates are lower for more diffuse interfaces [Figs. 2(a) and 2(e)]. The capillary forces dampen the development of the short-wavelength modes, which is illustrated by Fig. 2(b). The surface tension effect reduces the range of the unstable modes by the cutoff value $k_{c}=\sqrt{3 \delta \mathrm{Gr} / \mathrm{Ca}}$.

Dependencies on the interface thickness differ for the cases of zero and nonzero capillary numbers. If the capillary forces can be disregarded, i.e., $\mathrm{Ca}=0$, the curves obtained for the gradually decreasing values of the interface thickness converge to the sharp-interface results [Fig. 2(a)]. When $\mathrm{Ca} \neq 0$, i.e., the capillary effects are taken into account, a sole reduction of the interface thickness does not produce the behavior of a sharp interface. This can be easily explained by the fact that the surface tension is defined as the ratio between the capillary number and the interface thickness (22), and hence by decreasing the interface thickness we increase the capillary effect, which is revealed by stronger damping of the short-wavelength modes [Fig. 2(c)]. In order to obtain the sharp-interface behavior, the capillary number and the interface thickness must be simultaneously decreased, which is done in Figs. 2(d) and 2(f).

For the gravity-capillary waves developing at an interface separating two viscous liquids, both real and imaginary parts of $\omega$ are different from zero; these functions are plotted in Fig. 3. Again, all classical properties of the gravity-capillary waves can be reproduced. It can be shown that in the inviscid limit the phase speed is proportional to $k^{-1 / 2}$; i.e., longer (a) GCW

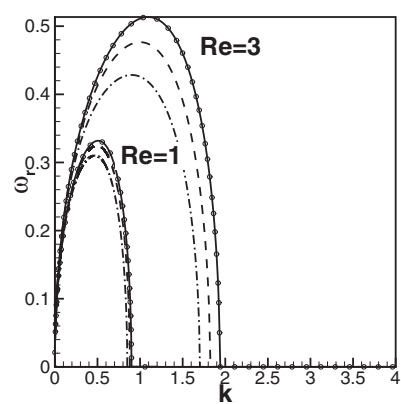

(c) GCW

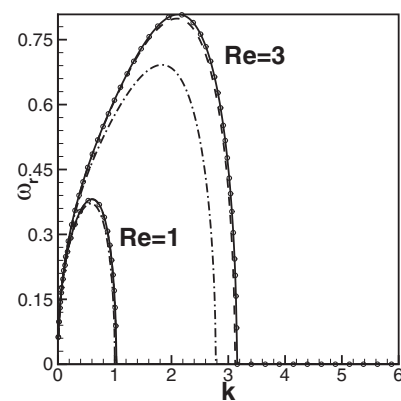

(b) GCW

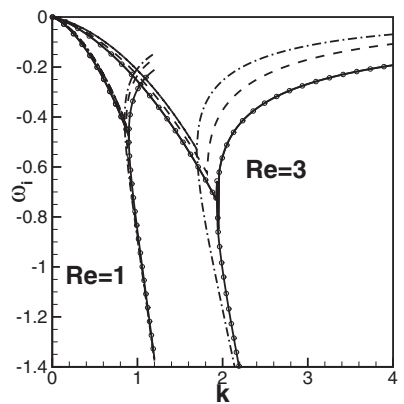

(d) GCW

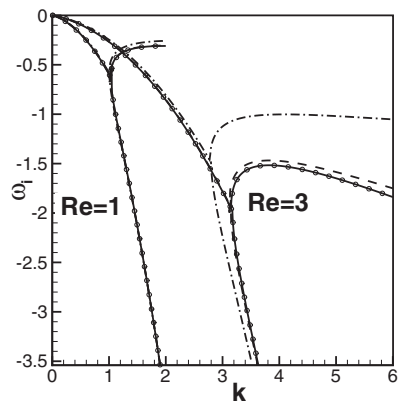

FIG. 3. Gravity-capillary waves at an immiscible interface. The real and imaginary parts of the frequency $\omega$ versus the wave number $k$ are shown for $\mathrm{Gr}=0.9$ and $\mathrm{Re}=1$ and $\mathrm{Re}=3$, and (a), (b) $\mathrm{Ca}=0$ and various interface thicknesses $(\delta=1$, dash-dot line; $\delta=0.5$, dashed line; and $\delta=0.01$, solid line); (c), (d) for a constant ratio $\mathrm{Ca} / \delta=1(\mathrm{Ca}=\delta=0.5$, dash-dot line; $\mathrm{Ca}=\delta=0.1$, dashed line; and $\mathrm{Ca}=\delta=0.01$, solid line). Symbol "o" marks the sharp-interface results. 
modes propagate faster. The waves are dampened by the viscous effect: Some modes start dissipating monotonically (with appearance of the creeping and viscous modes, totally similar to the waves at a sharp interface [6]), and hence loosing their wavelike behavior. One also sees that the capillarity primarily affects the development of the modes with shorter wavelengths.

Figure 4 depicts the eigenfunctions, i.e., the stream function profiles for the Rayleigh-Taylor instability and gravity-capillary waves. In the case of the Rayleigh-Taylor instability, the stream function is always purely real, and in the case of the waves, the eigenfunction is real in the inviscid case and has both real and imaginary parts in the viscous case. The stream functions are continuous, but their derivatives are discontinuous if the viscous effect is not taken into account. In the viscous case, both stream function and derivative are continuous. One can also note that the thickness of the eigenfunction is of order 10, irrelevant to the thickness of the interface (which is 0.01 for the shown results), and is only slightly different for the inviscid and viscous cases. The eigenfunction profiles do not depend on the value of the capillary number. (a) RT

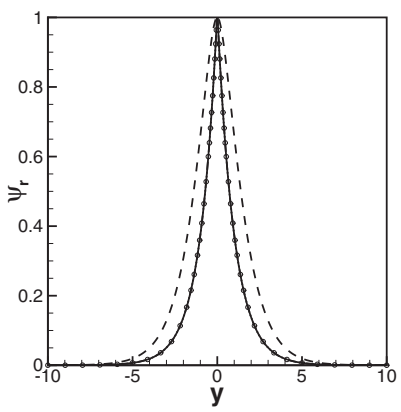

(c) GCW

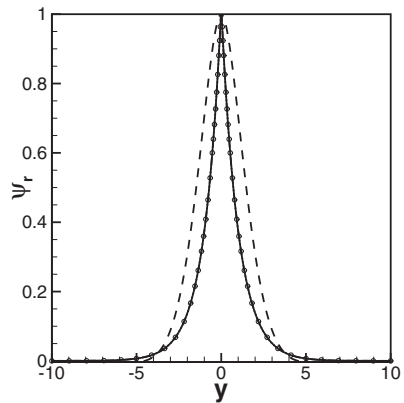

(b) RTI

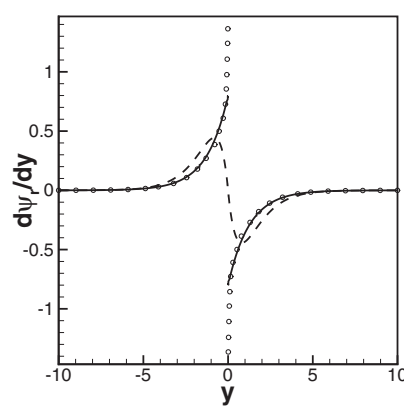

(d) GCW

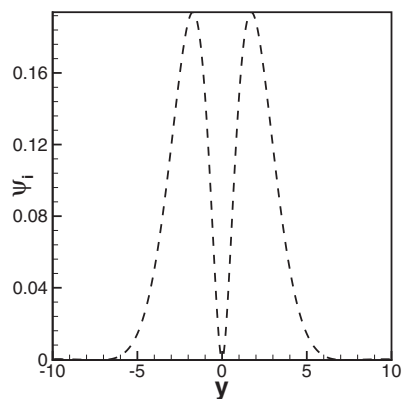

FIG. 4. Typical shapes of perturbations (eigenfunctions) induced at an immiscible interface at $k=1$. (a), (b) The real parts of the stream function and their first derivative are shown for the Rayleigh-Taylor instability (the imaginary part of the stream function is zero in this case). For $\mathrm{Ca}=0$, the growth rate is 0.740 (inviscid mode) and 0.405 (viscous mode); for $\mathrm{Ca}=0.05$, the growth rate is 0.630 (inviscid case). (c), (d) The real (c) and imaginary (d) parts of the stream function are plotted for the gravity waves. For $\mathrm{Ca}=0$, the eigenvalue is $\omega=(0,-0.74)$ (inviscid case) and $\omega=(0.511,-0.256)$ (viscous case); for $\mathrm{Ca}=0.05$, the eigenvalue is $\omega=(0,-0.630)$ (inviscid case). The eigenfunctions are plotted for $\mathrm{Gr}= \pm 1, \delta=0.01, \mathrm{Ca}=0$ for inviscid (solid lines) and viscous ( $\mathrm{Re}=3$, dashed lines) cases, and another eigenfunction for $\mathrm{Ca}=0.05$; inviscid case is marked by "o" symbols.
Finally, we wish to discuss the applicability of the phasefield approach. The interface is smeared and the new length scale, the interface thickness $\delta$, is introduced within this approach. Nevertheless, the thickness of a real phase boundary is usually very small, just several molecular layers, and hence the limit of a sharp interface is of paramount importance for the results of the phase-field theory. We found that a perfect agreement between the results obtained for the diffusive and sharp

(a)

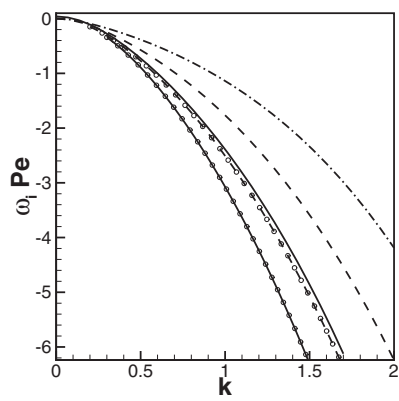

(b)

(c)

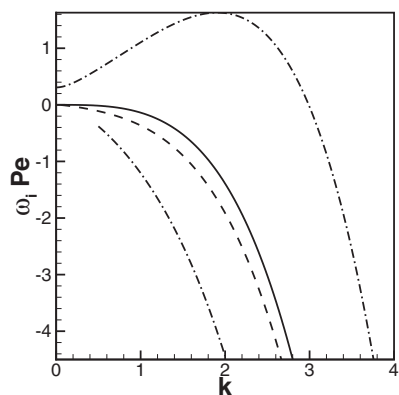

(e)
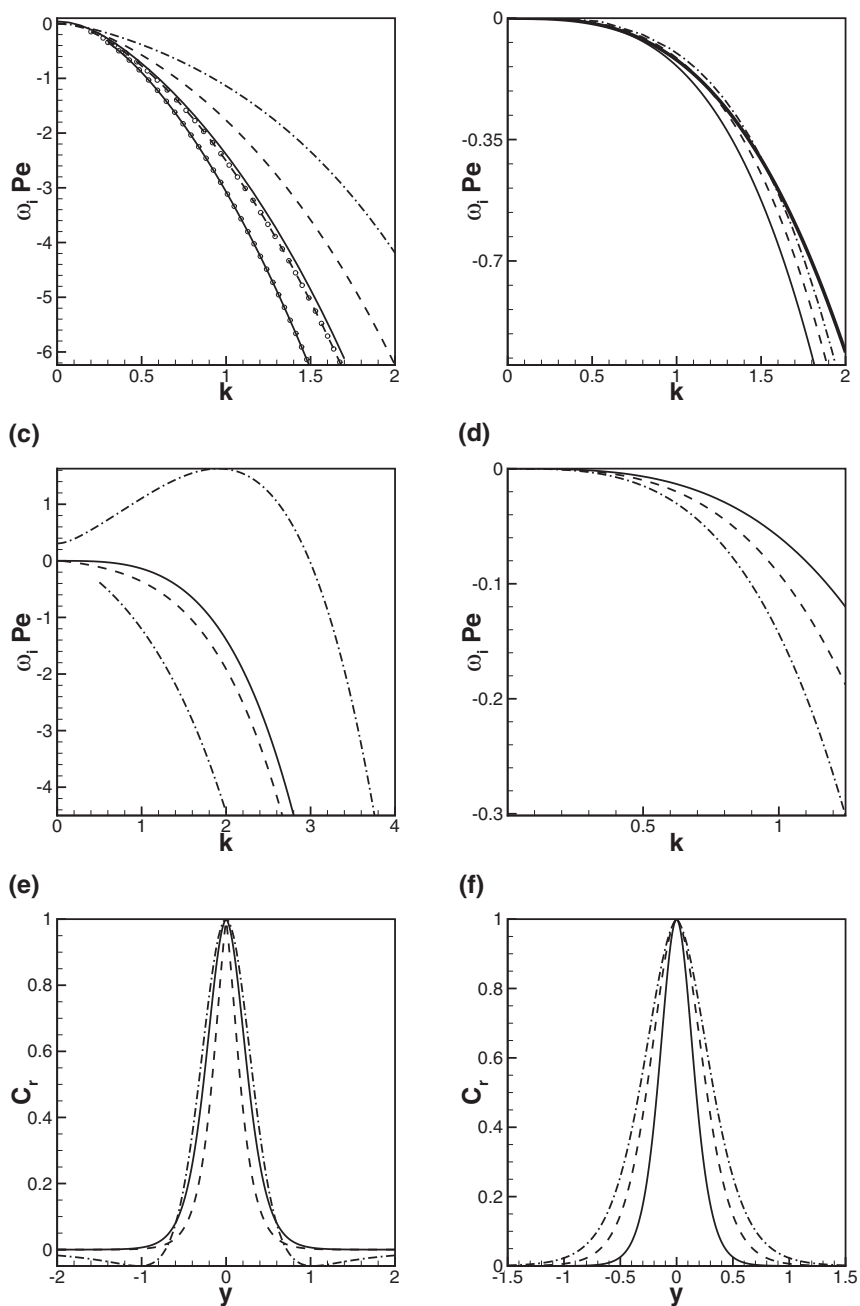

(d)

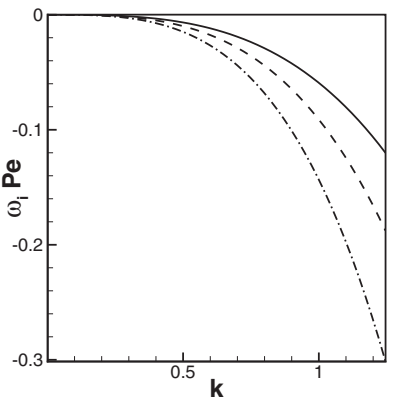

(f)

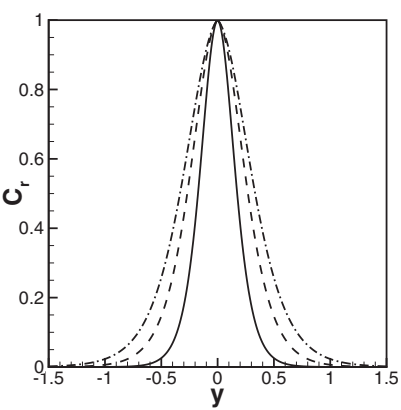

FIG. 5. (a)-(d) Eigenspectra are plotted for interfaces subject to thermodynamic disturbances. (a) $\mathrm{Ca}=0.05, \delta=1, A=0.5$ (solid line), $A=0.3$ (dashed line), and $A=0.1$ (dash-dot line) and $\delta=0.5$, $A=0.5$ (solid line with symbols) and $A=0.3$ (dashed line with symbols). (b) $\delta=\delta_{0}, \mathrm{Ca}=0.05$, and various $A<0(A=-0.1$, dash-dot line; $A=-0.3$, dashed line; $A=-0.5$, solid line; and thick solid line represents $-k^{3}$ curve); (c) $\mathrm{Ca}=0.05, A=-0.5$, and various $\delta\left(\delta=\delta_{0}=0.316\right.$, solid line; $2 \delta_{0}$, dash-dot-dot line; $0.8 \delta_{0}$, dashed line; $0.6 \delta_{0}$, dash-dot line); (d) $A=-0.4, \delta=\delta_{0}$, and various $\mathrm{Ca}(\mathrm{Ca}=0.07$, dash-dot line; 0.035, dashed line; and 0.01, solid line). (e), (f) Eigenfunctions are plotted for $k=1, A=-0.5$, (e) $\mathrm{Ca}=0.05$ and various $\delta\left[\delta=2 \delta_{0}\right.$, dash-dot line $\left(\omega_{i}=0.633\right) ; \delta=\delta_{0}$, solid line $\left(\omega_{i}=-0.155\right)$; and $\delta=0.5 \delta_{0}$, dashed line $\left(\omega_{i}=-0.436\right)$ ]; and (f) $\delta=\delta_{0}$ and various $\mathrm{Ca}\left[\mathrm{Ca}=0.02\right.$, solid line $\left(\omega_{i}=-0.186\right)$; $\mathrm{Ca}=0.05$, dashed line $\left(\omega_{i}=-0.079\right)$; and $\mathrm{Ca}=0.08$, dash-dot line $\left.\left(\omega_{i}=-0.053\right)\right]$. All eigenfunctions are purely real and normalized by their maximum values. 
interfaces is restricted by the inequality $k \delta \ll 1$. In Fig. 2(a), the numerical data obtained for the diffuse interfaces and the analytical data for a sharp boundary coincide while the wave number remains small, and such a matching deteriorates for larger $k$. For thin interfaces, e.g., with $\delta=0.001$, the numerical results closely follow the analytical curve; however, this remains true only within the shown range of the wave numbers. In order to obtain accurate results with the use of the phase-field approach, the interface thickness $\delta$ should be smaller than all other length scales, including the wavelength of perturbations $\lambda=2 \pi / k$, which one cannot guarantee for arbitrary perturbations. The surface tension and viscous effects introduce the cutoff wave number, $k_{c}$, limiting the range of the unstable modes in the case of the Raleigh-Taylor instability and the range of the modes with the periodic wavelike behavior in the case of the gravity-capillary waves. In this case, the condition on $\delta$ becomes $k_{c} \delta \ll 1$, and the accurate eigenvalue spectra may be obtained for interface thicknesses of order $\delta \sim 0.1-0.01$.

\section{THERMODYNAMIC STABILITY OF A MISCIBLE INTERFACE}

We now consider an opposite situation with neglected hydrodynamic flows, and hence with the mass transfer purely (a) RTI

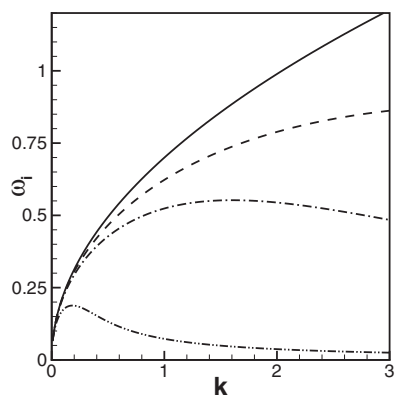

(c) RTI

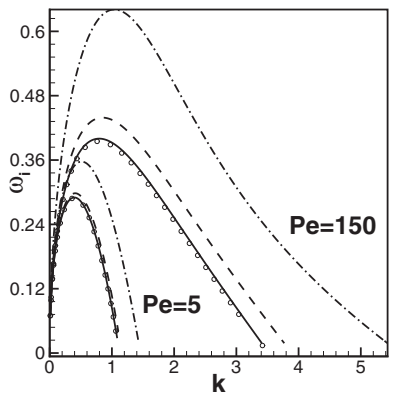

(b) RTI

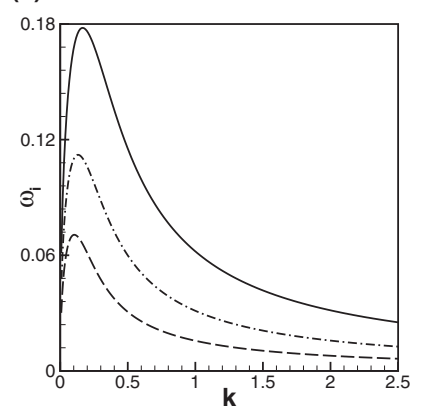

(d) RTI

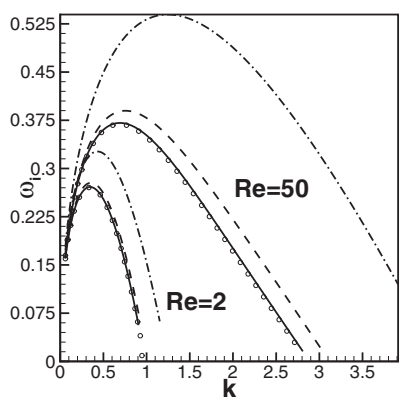

FIG. 6. Rayleigh-Taylor instability at a miscible interface between two inviscid [(a)-(d)] and viscous [(e), (f)] liquids. Capillary effects are disregarded. The growth rate $\omega_{i}$ versus the wave number $k$ for (a) $\mathrm{Gr}=-0.9, A=0.2$, and $\delta=0.001$, and various Peclet number $(\mathrm{Pe}=\infty$, solid line; $\mathrm{Pe}=100$, dashed line; $\mathrm{Pe}=25$, dashdot line; and $\mathrm{Pe}=1$, dash-dot-dot line); and (f) $\mathrm{Pe}=1, A=0.5$, $\delta=0.001$, and various Grashof numbers $(\mathrm{Gr}=-1$, solid line; $\mathrm{Gr}=-0.5$, dashed line; $\mathrm{Gr}=-0.25$, dash-dot line); (c) $\mathrm{Gr}=-1$, $A=-0.5, \mathrm{Re}=3, \mathrm{Pe}=5$ and $\mathrm{Pe}=150$ and various $\delta(\delta=0.1$, dashed line; $\delta=0.001$, solid line; $\circ$ marks $\delta=0.0001$ ); and (d) $\mathrm{Gr}=$ $-1, A=-0.5, \mathrm{Pe}=5, \operatorname{Re}=2$ and $\operatorname{Re}=50$, and various $\delta$ [as in (c)]. driven by diffusion. The full set of amplitude equations (26)-(28) is reduced to an equation written in terms of the concentration field,

$$
\begin{aligned}
& \mathrm{Ca} C^{i v}-\left(D_{0}+2 \mathrm{Ca} k^{2}\right) C^{\prime \prime}-2 D_{0}^{\prime} C^{\prime} \\
& \quad+\left[k^{2}\left(D_{0}+\mathrm{Ca} k^{2}\right)-D_{0}^{\prime \prime}-i \omega \mathrm{Pe}\right] C=0,
\end{aligned}
$$

where $D_{0} \equiv 2 A+12 C_{0}^{2}$ is the diffusion coefficient. We use this equation to investigate the linear instability of a heterogeneous miscible system with respect to thermodynamic perturbations.

The results of the numerical solution of eigenvalue problem (35) are depicted in Fig. 5. All eigenvalues were found to be purely imaginary. We see that the interface is always stable (all normal perturbations decay) for $A>0$. The stability is increased (the decay rates grow) for greater values of $A$ and smaller thicknesses $\delta$. If however $A<0$, the layer may be either stable or unstable which is determined by the interface thickness. Namely, all perturbations monotonically decay if the interface thickness is less than or equal to the equilibrium value, $\delta \leqslant \delta_{0}$, but the long-wavelength instability is observed if the interface thickness is greater than $\delta_{0}$, which is illustrated in Fig. 5(c). We also confirmed that when $\delta=\delta_{0}$, the decay rate follows the $k^{3}$ dependence for small values (a) RTI

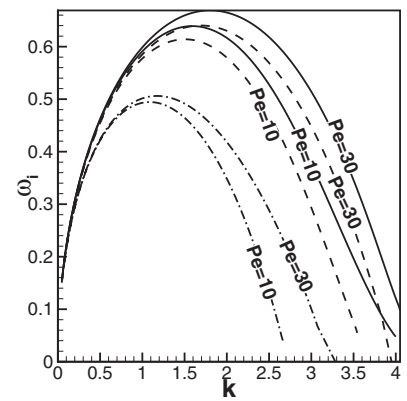

(c) RTI

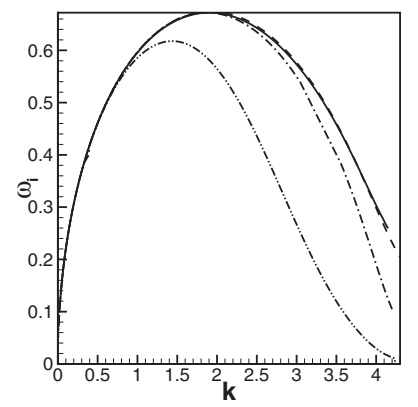

(b) RTI

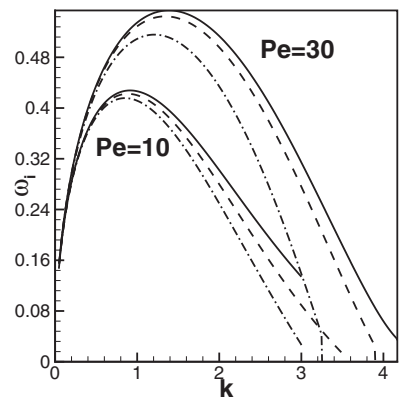

(d) RTI

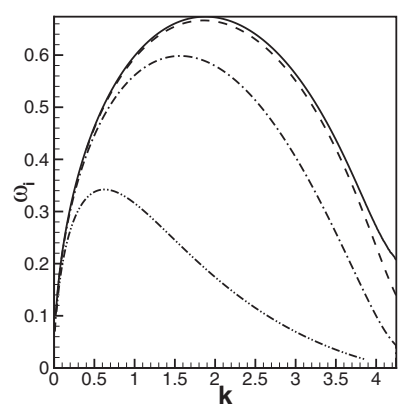

FIG. 7. Rayleigh-Taylor instability at a miscible interface. The growth rate $\omega_{i}$ versus the wave number $k$ is depicted for $\mathrm{Gr}=-1$, $\mathrm{Ca}=0.05, \delta=0.3$, (a) $A=-0.5, \mathrm{Pe}=10$ and $\mathrm{Pe}=30$, and various $\mathrm{Re}$ (inviscid result, solid line; $\mathrm{Re}=250$, dashed line; $\mathrm{Re}=50$, dashdot line); (b) $A=0.5, \mathrm{Pe}=10$ and $\mathrm{Pe}=30$, and various $\mathrm{Re}$ [lines as in (c)]; (c) $A=-0.5, \operatorname{Re}=10$, and various Pe (immiscible case, solid line; $\mathrm{Pe}=100$, dashed line; $\mathrm{Pe}=10$, dash-dot line; $\mathrm{Pe}=1$, dash-dot-dot line); (d) $A=0.5, \mathrm{Re}=10$, and various $\mathrm{Pe}$ (immiscible case, solid line; $\mathrm{Pe}=250$, dashed line; $\mathrm{Pe}=50$, dash-dot line; $\mathrm{Pe}=$ 10, dash-dot-dot line). 
of $k$ (and one sees that for $\delta<\delta_{0}$ the decay rate changes even faster). The effect of the capillarity on the growth rates is shown in Fig. 5(d), where one sees that the modes with long wavelengths remain virtually unaffected, but the modes with shorter wavelengths decay faster if the value of $\mathrm{Ca}$ is larger.

One can also note that the results in Fig. 5 are plotted in terms of the product $\omega_{i} \mathrm{Pe}$, and hence the decay/growth rates of pure thermodynamic perturbations would be rather small for larger Pe numbers.

Figures 5(e) and 5(f) depict the typical shapes of the eigenfunctions, $C_{r}(z)$ (the imaginary part was found to be zero). The eigenfunctions shown are normalized by their maximum values, located in the middle of the layer. Figure 5(e) shows the eigenfunctions for $A<0$. One sees that the thickness of the eigenfunction diminishes for smaller values of $\delta$. The widths of the eigenfunction depicted in Figs. 5(e) and 5(f) is considerably smaller (by an order of magnitude) compared to the widths of the purely hydrodynamic modes shown in Figs. 4. One can also notice in Fig. 5(f) that the widths of the eigenfunctions depicted in Fig. 5(f) diminish if $\mathrm{Ca}$ becomes smaller. Moreover, we found that the eigenvalue problem (35) has no nontrivial solutions for $\mathrm{Ca}=0$. (a) RTI

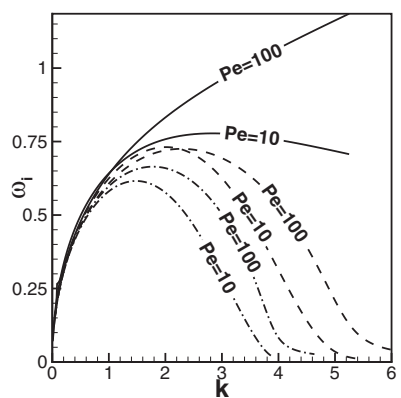

(c) RTI

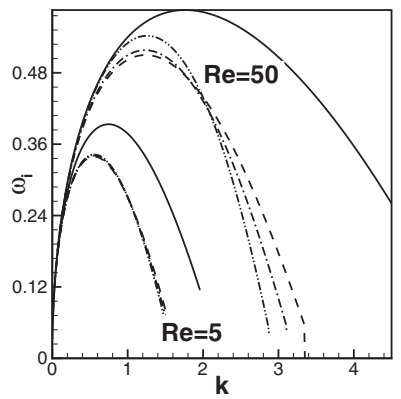

(b) RTI

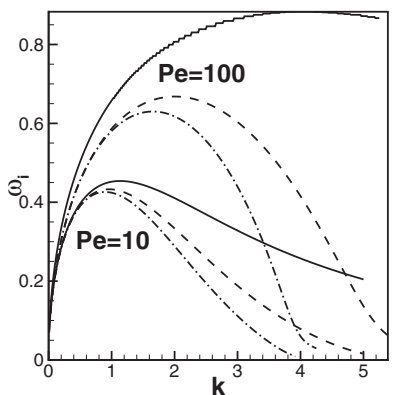

(d) RTI

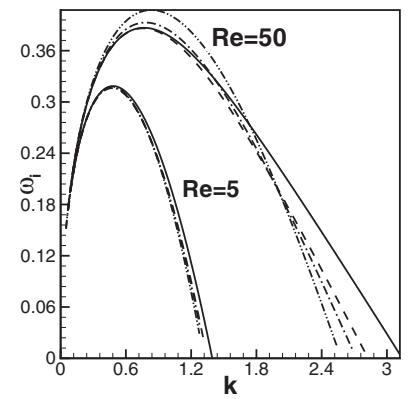

FIG. 8. Rayleigh-Taylor instability at a miscible interface. The growth rate $\omega_{i}$ versus the wave number $k$ is depicted for $\mathrm{Gr}=-1$, (a) $\mathrm{Pe}=10$ and $\mathrm{Pe}=100, A=-0.5, \delta=0.3$ and various capillary numbers $(\mathrm{Ca}=0$, solid line; $\mathrm{Ca}=0.03$, dashed line; $\mathrm{Ca}=0.06$, dash-dot line), (b) $\mathrm{Pe}=10$ and $\mathrm{Pe}=100, A=0.5, \delta=0.3$ [lines as in (a)]; (c) $\mathrm{Pe}=10, A=-0.5, \delta=0.05$, and various $\mathrm{Ca}(\mathrm{Ca}=0$, solid line; $\mathrm{Ca}=0.02$, dashed line; $\mathrm{Ca}=0.04$, dash-dot line; and $\mathrm{Ca}=0.08$, dash-dot-dot line); (d) $\mathrm{Pe}=10, A=0.5, \delta=0.05$ [lines as in (c)]. (a) and (b) depict the inviscid results; (c) and (d) depict the viscous results for $\operatorname{Re}=5$ and $\operatorname{Re}=50$.

\section{STABILITY OF A MISCIBLE INTERFACE SUBJECT TO BOTH THERMO- AND HYDRODYNAMIC PERTURBATIONS}

\section{A. Rayleigh-Taylor instability}

The aim of this section is to investigate the simultaneous actions of the small hydro- and thermodynamic perturbations on the stability of the flat interface. We however saw that the thermodynamic modes do not exist when the capillary effects are completely disregarded. In addition, in this case the sharp interface behavior could be revealed by simple gradual reduction of the interface thickness as shown in Sec. III. (a) RTI

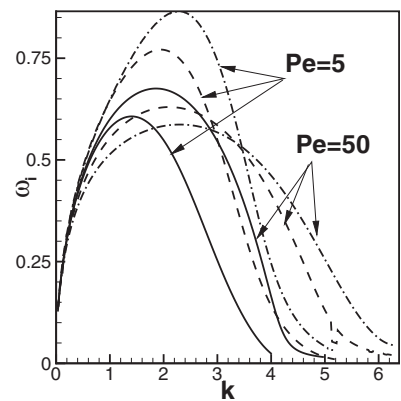

(c) RTI

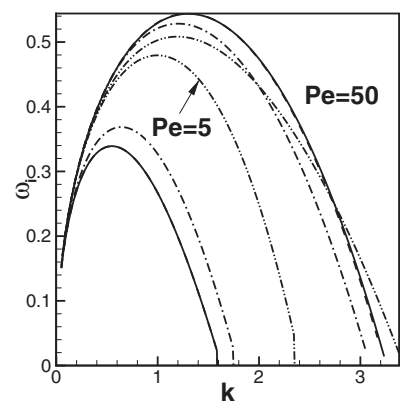

(e) RTI

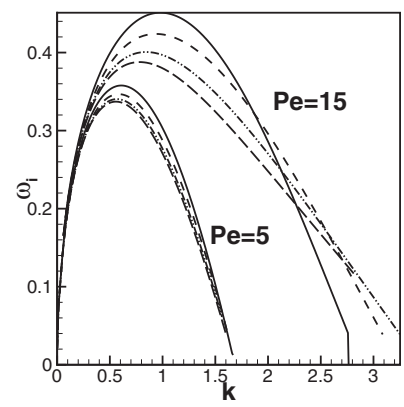

(b) RTI

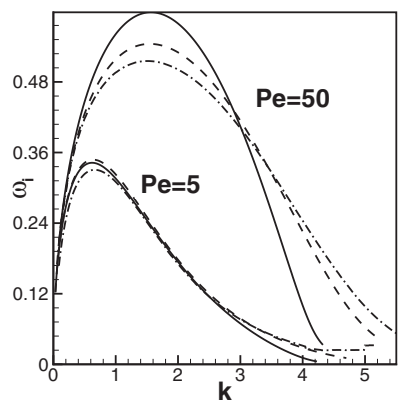

(d) RTI

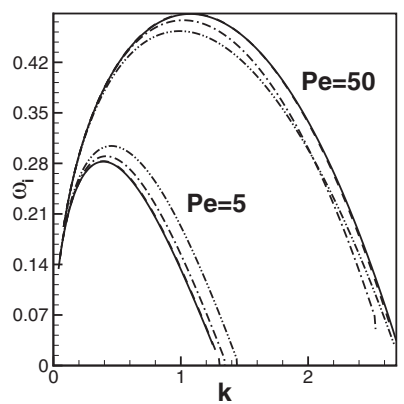

(f) RTI

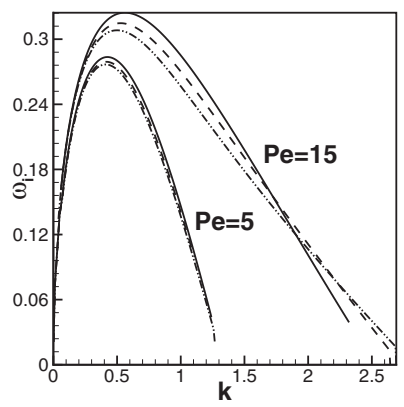

FIG. 9. Rayleigh-Taylor instability at a miscible interface. The growth rate $\omega_{i}$ versus the wave number $k$ is depicted for $\mathrm{Gr}=-1$, $\mathrm{Ca}=0.05, \mathrm{Pe}=5$ and $\mathrm{Pe}=50$, (a) $A=-0.5$ and various interface thicknesses ( $\delta=0.9$, dash-dot line; $\delta=0.6$, dashed line; $\delta=0.3$, solid line); (b) $A=0.5$ [lines as in (a)]; (c) $A=-0.5, \operatorname{Re}=10$, and various $\delta$ ( $\delta=0.001$, solid line; $\delta=0.01$, dashed line; $\delta=0.1$, dash-dot line; and $\delta=0.3$, dash-dot-dot line); (d) $A=0.5$, Re $=10$ [lines as in (c)]. (a), (b) depict the inviscid results. (e), (f) $\mathrm{Gr}=$ $-1, \operatorname{Re}=10, \mathrm{Pe}=5$ and $\mathrm{Pe}=15$, (e) $A=-0.5$; (f) $A=0.5$; and different capillary numbers and interface thicknesses for $\mathrm{Ca} / \delta=1$ $(\mathrm{Ca}=\delta=0.02$, solid line; $\mathrm{Ca}=\delta=0.04$, dashed line; $\mathrm{Ca}=\delta=$ 0.08 , dash-dot line). 
(a) RTI

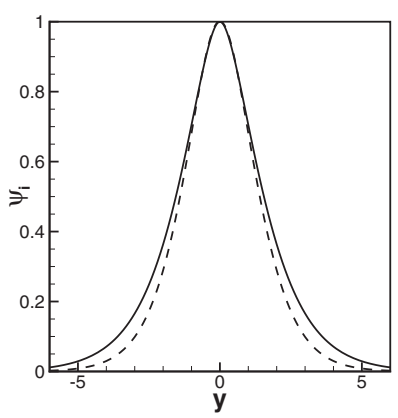

(c) RTI

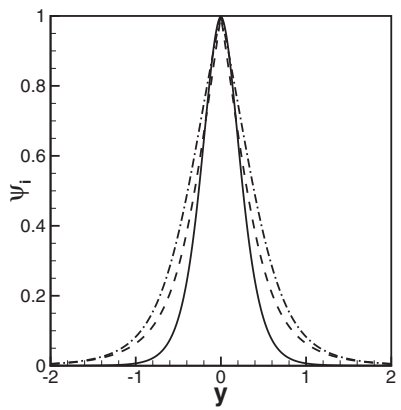

(b) RTI

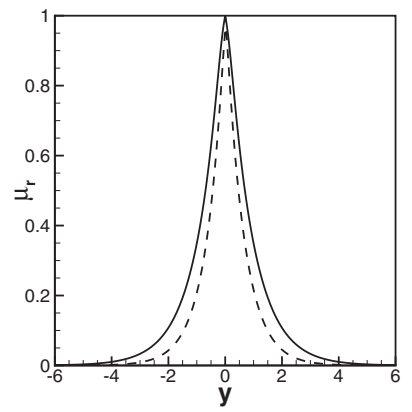

(d) RTI

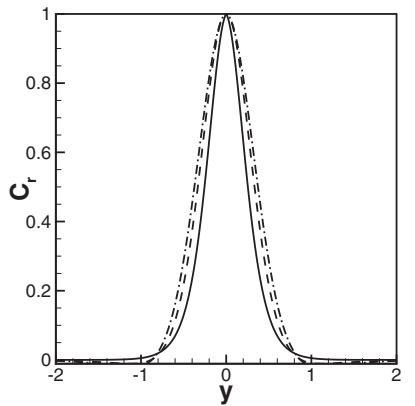

FIG. 10. The eigenfunctions for the Rayleigh-Taylor instability developed at a miscible interface. (a), (b) $\mathrm{Ca}=0$, profiles of the stream function (a) and chemical potential (b) are shown for the inviscid (solid lines) and viscous ( $\mathrm{Re}=10$, dashed lines) cases. The results are shown for $\mathrm{Gr}=-1, k=1, \mathrm{Pe}=5, \delta=0.1$, and $A=0.5$. The growth rate is $\omega_{i}=0.306$ in the inviscid case and $\omega_{i}=0.176$ in the viscous case. (c), (d) $\mathrm{Ca}=0.05$, profiles of the imaginary part of stream function (c) and the real part of concentration (d) are shown for the interfaces between two inviscid liquids; the interface thicknesses are 0.3 (solid line), 0.6 (dashed line), and 0.9 (dash-dot line); other parameters are $\mathrm{Gr}=-1, \mathrm{Pe}=10, A=-0.5, k=2$. The growth rates are $0.778(\delta=0.9), 0.770(\delta=0.6)$, and $0.548(\delta=0.3)$.

The eigenvalue spectra obtained for the Rayleigh-Taylor instability in the case of neglected capillary effects are shown in Fig. 6. One can notice that the role of diffusion is reduced to additional dissipation [see Fig. 6(a)], in many ways, similar to the effect exerted by viscosity. We also found that the results of immiscible interfaces are recovered for very large Peclet numbers. The similarity between the viscous and diffusive effects can be already deduced from the amplitude equations in which the viscous and diffusive terms are quite similar, especially if $\mathrm{Ca}=0$. Thus, in the case of $y \rightarrow \infty$, the viscous and diffusive terms will be different by only the factors of $\mathrm{Re}$ and $\mathrm{Pe} / D_{0}$. We also found that the cumulative action of viscosity and diffusivity is able to completely stabilize the growth of the modes of shorter wavelengths [Figs. 6(c) and 6(d)]; however, separately, neither viscosity [Fig. 2(e)] nor diffusivity [Fig. 6(a)] were capable of doing this.

In the case of $\mathrm{Ca}=0$, we found that the variation of the parameter $A$ does not lead to any qualitative changes in the stability results, and the increase in $A$ simply results in stronger damping. Figure 6(b) shows how the growth rates of perturbations depend on the Grashof number, as one sees the instability is increased upon the increase in $\mathrm{Gr}$, but the positions of the fastest growing modes remain almost unchanged.

(a) GW

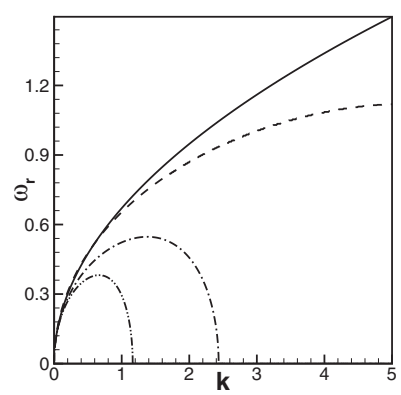

(c) GW

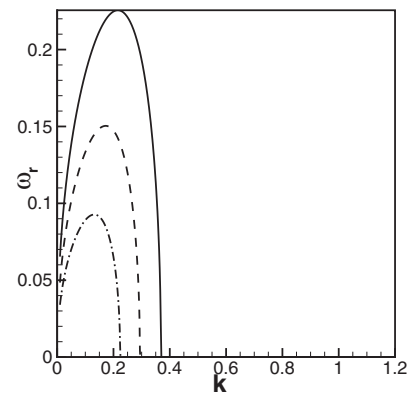

(e) GW

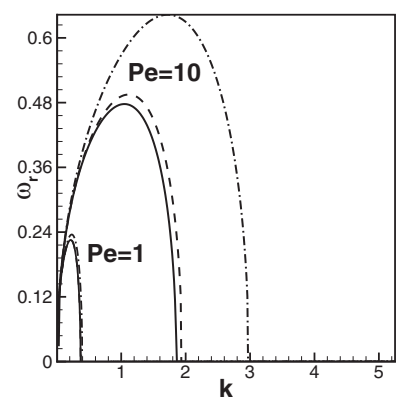

(b) GW

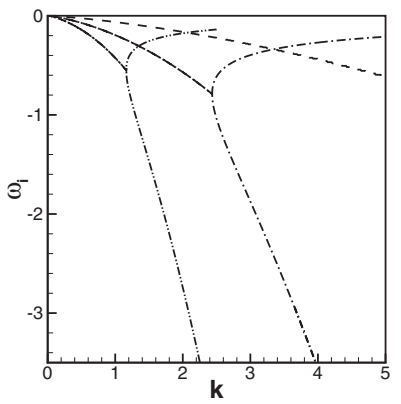

(d) GW

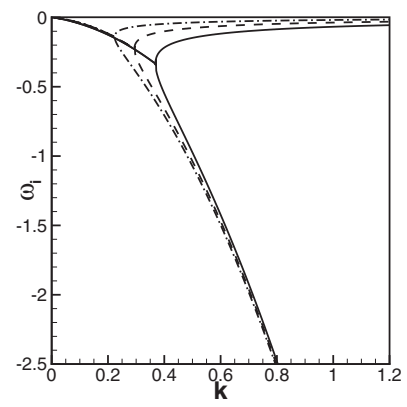

(f) GW

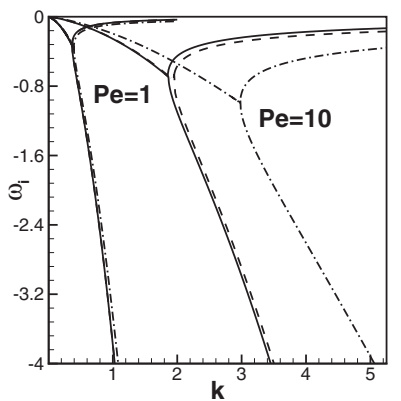

FIG. 11. Dispersion relations and decay rates for the gravity waves on a miscible interface between two inviscid liquids. Capillary terms are neglected. The data are shown for (a), (b) $\mathrm{Gr}=0.9$, $A=0.2, \delta=0.001$, and various Peclet numbers ( $\mathrm{Pe}=5$, dash-dotdot line; $\mathrm{Pe}=15$, dash-dot line; dashed line $\mathrm{Pe}=100$; immiscible case, solid line); (c), (d) $\mathrm{Pe}=1, A=0.2, \delta=0.001$, and various Grashof numbers $(\mathrm{Gr}=-0.9$, solid line; $\mathrm{Gr}=-0.5$, dashed line; $\mathrm{Gr}=-0.25$, dash-dot line); and (e), (f) $A=0.2, \mathrm{Pe}=1$ and $\mathrm{Pe}=$ $10, \mathrm{Gr}=0.9$, and various interface thicknesses $(\delta=0.05$, dash-dot line; $\delta=0.01$, dashed line; and $\delta=0.001$, solid line).

Figures 6(c) and 6(d) show how the growth rate of perturbations depends on the value of the interface thickness, illustrating that the curves converge to the limit of a sharp interface when $\delta$ tends to zero. Similar to immiscible interfaces, the convergence is perfect for the shown range of $k$, but may deteriorate for the modes with larger wave numbers. The stabilization of the short-wavelength modes by the combined actions of viscosity and diffusivity relaxes the restriction on the value of the interface thickness. If one is interested in the unstable part of the eigenspectrum, or in the fastest growing modes, then the thickness of the interface can be taken as $\delta k_{c}(\mathrm{Re}, \mathrm{Pe}) \ll 1$, and the instability of a sharp interface will be accurately reproduced.

Next, we consider the solution of the full amplitude equations (26)-(28). The obtained numerical solution first 
allows us to confirm that the viscous force introduces the dissipation as usual, which is illustrated in Figs. 7(a) and 7(b). The inviscid result can be recovered by taking the Reynolds number large enough. The effect of diffusivity is also reduced to dissipation as illustrated in Figs. 7(c) and 7(d).

The capillary forces are also known to suppress the growth of the short-wavelength modes. The strength of the capillary effect is defined by both the capillary number and the interface thickness. Figure 8 shows how the results depend on the capillary number. One sees that the increase of the capillary force results in expectable reduction of the range of unstable modes and reduces the growth rates, which generally coincide with our earlier observations for immiscible interfaces.

Figure 9 illustrates the eigenspectra for the interfaces of different thicknesses. The curves are obtained for the inviscid and viscous cases at two different Peclet numbers. Here the effects of viscosity, diffusivity, and capillarity interact. In particular, the combined action of the viscous and diffusive dampings can make the capillary action on the interface stability negligible. One can notice that the decrease in the interface thickness (hence the increase in the surface tension) does not always give us the expected suppression of the modes with shorter wavelengths. This means that the efficiencies of the viscous, diffusive, and capillary mechanisms all depend on the interface thickness, but these three dependencies are different, so the change in the interface thickness leads sometimes to an unexpected behavior.

Figures 9(e) and 9(f) show the curves obtained for the different capillary numbers and interface thicknesses that are (a) GCW

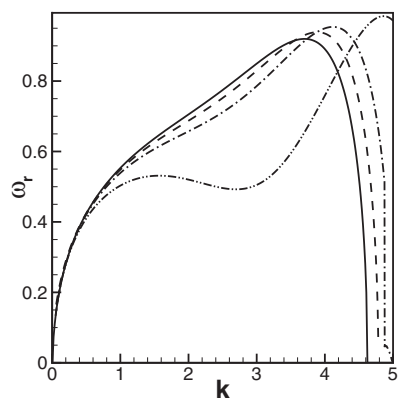

(c) GCW

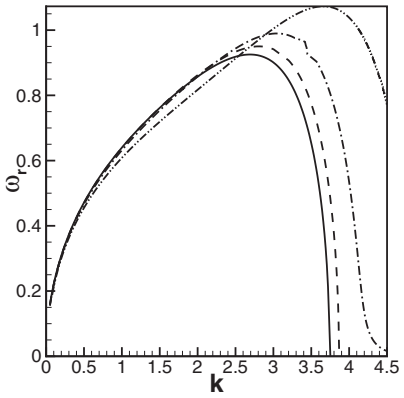

(b) GCW

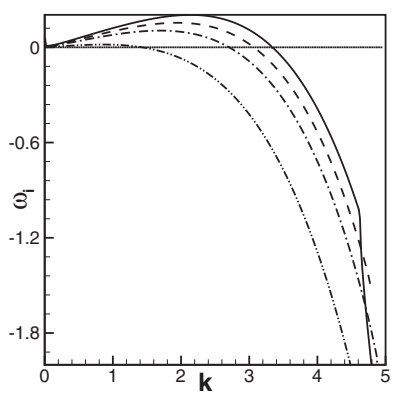

(d) GCW

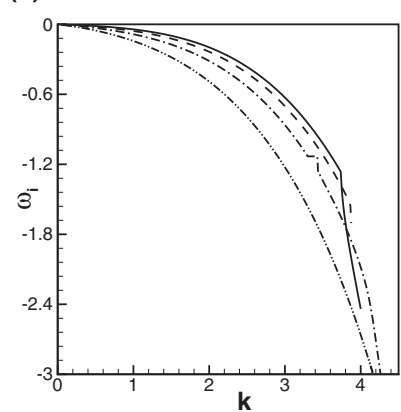

FIG. 12. Dispersion relations and decay rates for the gravitycapillary waves on a miscible interface. The curves are obtained for $\mathrm{Gr}=1, \mathrm{Ca}=0.04, \mathrm{Pe}=10, A=-0.5$, (a), (b) $\delta=2 \delta_{0} \approx 0.57$ (inviscid case, solid line; $\operatorname{Re}=100$, dashed line; $\operatorname{Re}=30$, dash-dot line; $\operatorname{Re}=10$, dash-dot-dot line); (c), (d) $\delta=0.25$ and various $\operatorname{Re}$ (inviscid case, solid line; $\mathrm{Re}=70$, dashed line; $\mathrm{Re}=30$, dash-dot line; $\operatorname{Re}=10$, dash-dot-dot line). changed so the ratio $\mathrm{Ca} / \delta$ remains constant. We see that the convergence to the limiting sharp-interface behavior can be achieved this way.

Finally, the typical shapes of eigenfunctions are depicted in Fig. 10 for both cases of $\mathrm{Ca}=0$ and $\mathrm{Ca} \neq 0$. The plotted eigenfunctions have very different $y$ widths. In the case of $\mathrm{Ca}=0$, the typical thickness of the eigenfunction is of order 10 , irrelevant to the thickness of the interface itself, and just slightly dependent on the values of the Reynolds number. In the case of $\mathrm{Ca} \neq 0$, one notices that the perturbation $y$ profile becomes more compact. In the former case $(\mathrm{Ca}=0)$, the eigenfunction profile is more similar to the one depicted in Fig. 4, i.e., to the purely hydrodynamic mode, while in the latter case $(\mathrm{Ca} \neq 0)$, the eigenfunction looks similar to Figs. 5(e) and 5(f), i.e., to the purely thermodynamic modes. These observations confirm that the evolution is driven by hydrodynamic modes in the case of $\mathrm{Ca}=0$, and thermodynamic effects become dominating for $\mathrm{Ca} \neq 0$.

\section{B. Gravity-capillary waves}

Similar to the previous section, our analysis for the gravitycapillary waves starts from the case of the pure gravity waves, i.e., when $\mathrm{Ca}=0$. Figures 11(a) and 11(b) illustrate the similarity of the effect exerted by diffusion to the viscous damping. Diffusivity introduces an additional dissipation mechanism, and similarly to the viscosity effect there appears the cutoff wave number that limits the range of the modes exhibiting the wavelike behavior. The solution for the larger wave numbers (a) GCW

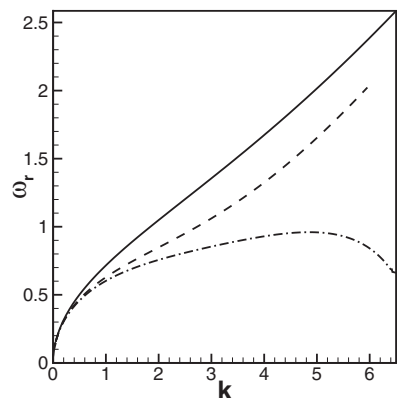

(c) GCW

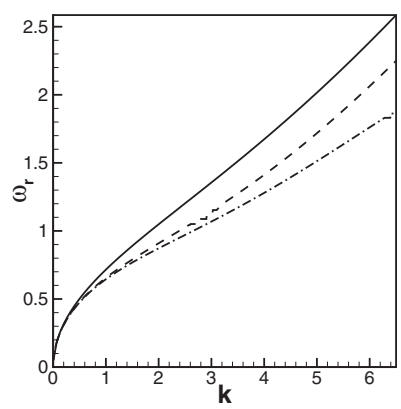

(b) GCW

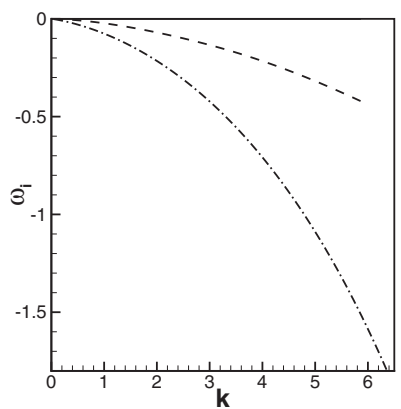

(d) GCW

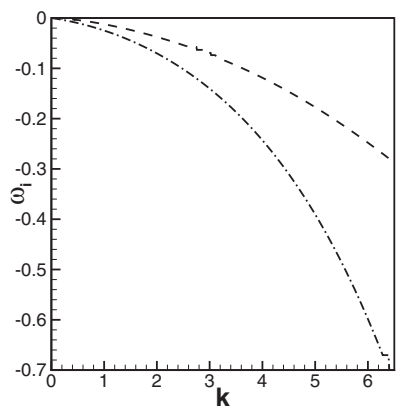

FIG. 13. Dispersion relations and decay rates for the gravitycapillary waves on the surface of a miscible interface separating two inviscid liquids are shown for various Peclet numbers (immiscible case, solid line; $\mathrm{Pe}=500$, dashed line; and $\mathrm{Pe}=50$, dash-dot line). The results are shown for $\mathrm{Gr}=1, \mathrm{Ca}=0.015, \delta=0.1$, (a), (b) $A=-0.5$, and (c), (d) $A=0.5$. 
becomes nonunique, as the eigenvalues have two possible imaginary parts. At the point of bifurcation, the real part of the eigenvalue becomes equal zero; hence these solutions describe quickly and monotonically dissipating perturbations. We also notice that the results for immiscible interfaces can be recovered by taking very large Peclet numbers, and, in the limit of small wave numbers, the phase speed of the perturbations follows the classical $k^{-1 / 2}$ dependence.

Figures 11(c) and 11(d) show that the increase in the value of the Grashoff number results in the increase of the phase speed of perturbations; however, the damping rate remains almost unaffected. Without the capillary terms, the waves are purely driven by the gravity force, and hence disappear when $\mathrm{Gr}$ tends to zero. In the case $\mathrm{Ca}=0$, an increase in the value of the parameter (a) GCW

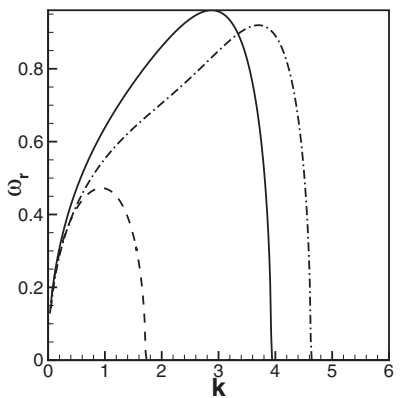

(c) GCW

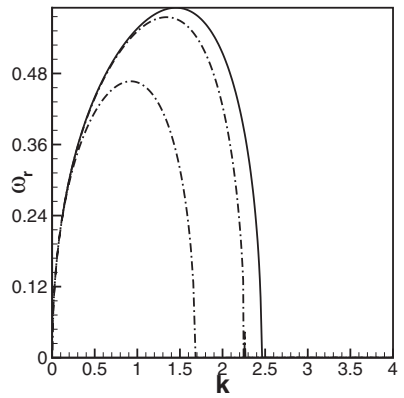

(e) GCW

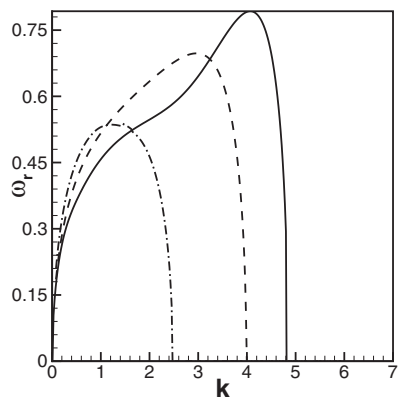

(b) GCW

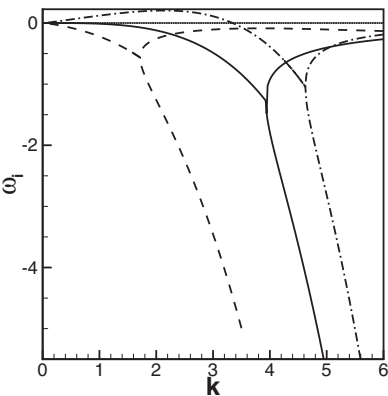

(d) GCW

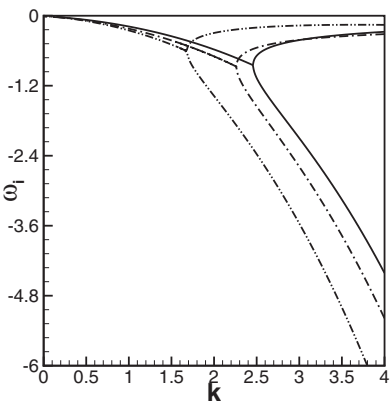

(f) GCW

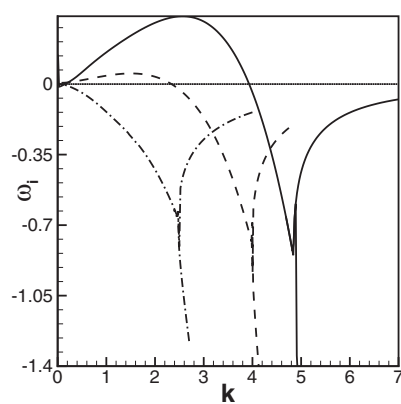

FIG. 14. Dispersion relations and decay rates for the gravitycapillary waves on the surface of a miscible interface. The results are shown for $\mathrm{Gr}=1, \mathrm{Ca}=0.04, \mathrm{Pe}=10$, (a), (b) $A=-0.5$, and various interface thicknesses $\left(\delta=\delta_{0}\right.$, sold line; $\delta=\delta_{0} / 3$, dashed line; and $\delta=2 \delta_{0}$, dash-dot line); and (c), (d) $A=0.5$ and various $\delta(\delta=0.1$, solid line; $\delta=0.2$, dashed line; and $\delta=0.4$, dash-dot line). (e), (f) The results are shown for $\mathrm{Gr}=1, \mathrm{Pe}=5, \mathrm{Ca}=0.04$, $\delta=0.85$, and $A=-0.5$ (solid line), $A=-0.3$ (dashed line), and $A=-0.1$ (dash-dot line).
$A$ leads to an increase of the diffusion coefficient and hence to a stronger damping of the waves. Figures 11(e) and 11(f) show that the curves quickly converge to a sharp-interface limit if the interface thickness $\delta$ tends to zero.

Next, we discuss the dispersion relations obtained for the gravity-capillary waves defined by the full amplitude equations (26)-(28). We start from Fig. 12 which confirms that viscosity generally continues to play its classical dissipative role. However, one also sees that when and $A<0$ and $\delta>\delta_{0}$ [Figs. 12(a) and 12(b)], there appears the long-wave instability, obviously driven by the thermodynamic instability described in Sec. IV. The long-wave instability disappears if $\delta<\delta_{0}$, as seen in Figs. 12(c) and 12(d). Figure 13 shows that the inclusion of diffusivity also introduces an additional damping to the system.

Figures 14(a)-14(d) show the changes in the dispersion relations associated with the variations of the interface thickness. The dependencies differ for the positive and negative values of $A$, and again the most interesting results are observed for $A<0$, when the dynamics of thicker interfaces (with $\delta>\delta_{0}$ ) exhibits the development of the long-wave instability. This effect is illustrated in more detail in Figs. 14(e) and 14(f). Figures 14(a)-14(d) also show that the thinner interfaces are obviously characterized with stronger interfacial tensions, and hence exhibit stronger suppression of the short-wavelength modes.

Figure 15 shows the dispersion relations for the gravitycapillary waves for the various values of the capillary number (a) GCW

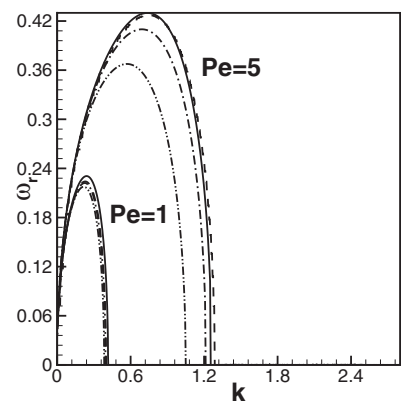

(c) GCW

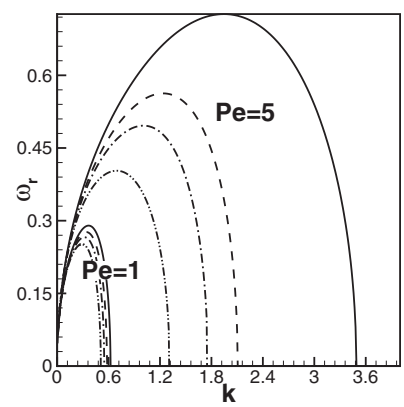

(b) GCW

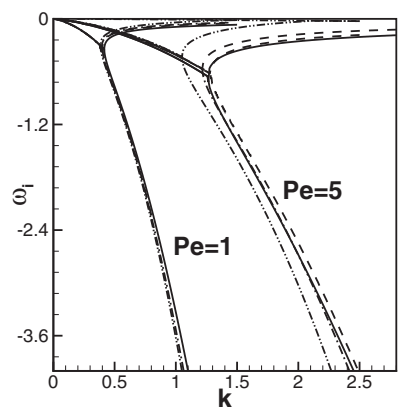

(d) GCW

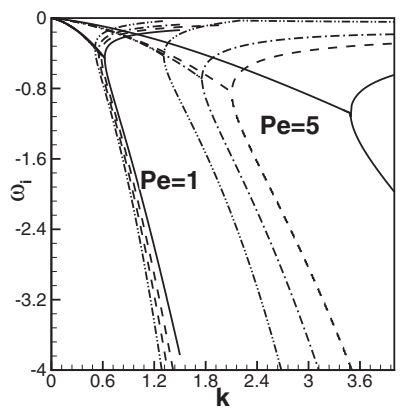

FIG. 15. Dispersion relations and decay rates for the gravitycapillary waves on a miscible interface separating two inviscid liquids. The results are shown for $\mathrm{Gr}=-0.9, \delta=0.1, \mathrm{Pe}=1$ and $\mathrm{Pe}=5$, (a), (b) $A=0.3$; (c), (d) $A=-0.3$. The curves are plotted for different $\mathrm{Ca}$ numbers: $\mathrm{Ca}=0$, solid line; $\mathrm{Ca}=0.015$, dashed line; $\mathrm{Ca}=0.03$, dash-dot line; and $\mathrm{Ca}=0.09$, dash-dot-dot line. 
also for the positive and negative values of $A$. One sees that the increase in the surface tension reduces the range of the modes with the wavelike behavior (i.e., the modes showing oscillatory decay). So the waves with shorter wavelengths are suppressed; however, the damping rates of other waves is just slightly affected by the increase of the capillary terms.

In Fig. 14 we saw that the properties of the interface are changed by varying the interface thickness, and the gradual reduction of the interface thickness does not produce the sharp interface behavior. Such a limiting behavior can however be obtained by the simultaneous reduction of the capillary number and interface thickness, so that the ratio $\mathrm{Ca} / \delta$ remains constant, which is illustrated in Fig. 16.

Finally, Fig. 17 shows the typical shapes of perturbations. The profiles are just slightly dependent on the value of the Reynolds and Peclet numbers. The widths of the eigenfunctions obtained for $\mathrm{Ca}=0$ [Figs. 17(a) and 17(b)] point out their similarity to the purely hydrodynamic modes of Fig. 4, and hence the development of such perturbations is driven by the hydrodynamic terms. In the case of $\mathrm{Ca} \neq 0$, the $y$ width of eigenfunctions depends on the interface thickness $\delta$, so for thicker interfaces the thickness of the eigenfunction increases. Nevertheless, the widths of the shown eigenfunctions point out their similarity to the thermodynamic modes depicted in Figs. 5(e) and 5(f), which means that the evolution in this case is dominated by the thermodynamic effects. (a) GCW

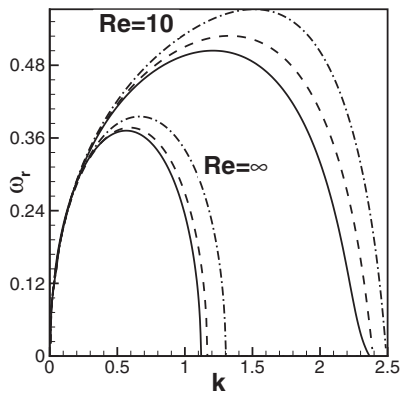

(c) GCW

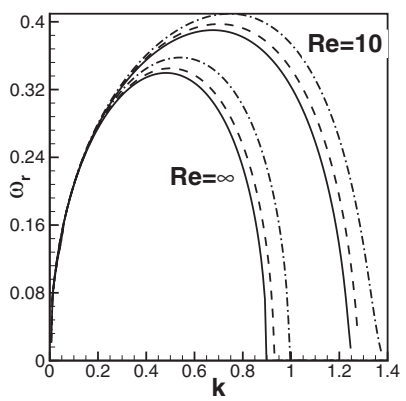

(a) GCW

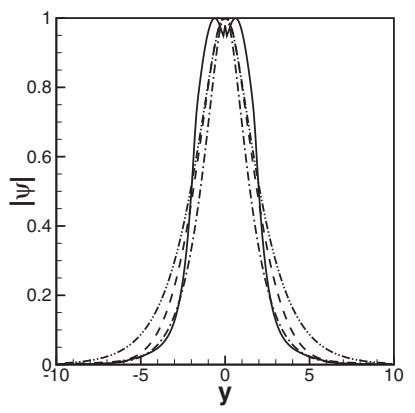

(e) GCW

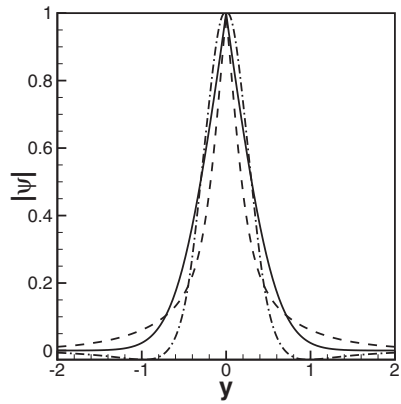

(b) GCW

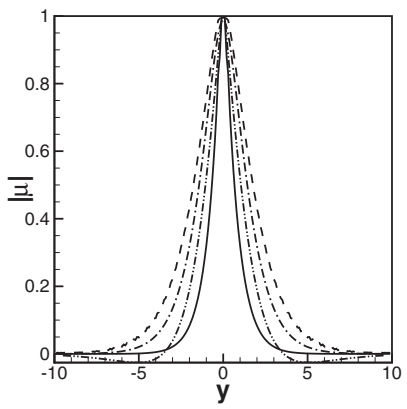

(f) GCW

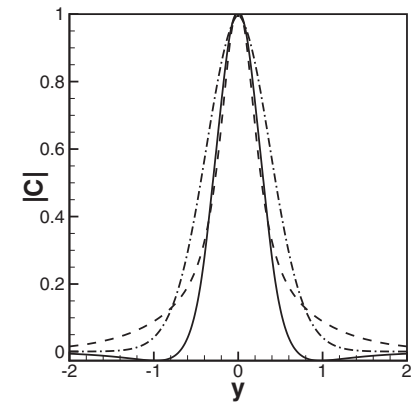

FIG. 17. The eigenfunctions are plotted for the gravity-capillary waves at a miscible interface. (a), (b) The capillary effects are disregarded. The moduli of the stream function (a) and chemical potential (b) are shown for $k=2, \mathrm{Gr}=1, A=0.5, \delta=0.1$, for the inviscid and viscous cases. Solid line depicts the results for $\mathrm{Pe}=15$, inviscid case; dashed line for $\mathrm{Pe}=5$, inviscid case; dash-dot line for $\mathrm{Pe}=15$ and $\mathrm{Re}=10$; and dash-dot-dot line for $\mathrm{Pe}=5$ and $\mathrm{Re}=10$. The eigenvalues are $\omega=(0.407,-0.542)(\mathrm{Pe}=5)$ and $\omega=(0.561,-0.275)(\mathrm{Pe}=15)$ for the viscous case and $\omega=$ $(0.382,-0.444)(\mathrm{Pe}=5)$ and $\omega=(0.592,-0.187)(\mathrm{Re}=15)$ for the inviscid case. (e), (f) The moduli of stream function (e) and concentration (f) are plotted for $k=1.75, \mathrm{Pe}=5, \mathrm{Gr}=-1, \mathrm{Ca}=$ 0.04, $A=-0.3$, and for $\delta=3 \delta_{0}$ (dash-dot line), $\delta=\delta_{0}$ (solid line), and $\delta=\delta_{0} / 2$ (dashed line). The eigenfunctions correspond to the eigenvalues of $\omega=(0.61,0.005) ;\left(\delta=3 \delta_{0}\right), \omega=(0.752,-0.193)$ $\left(\delta=\delta_{0}\right)$, and $\omega=(0.61,-0.435)\left(\delta=\delta_{0} / 2\right)$. The functions are normalized by the maximum values of the moduli. (d) GCW

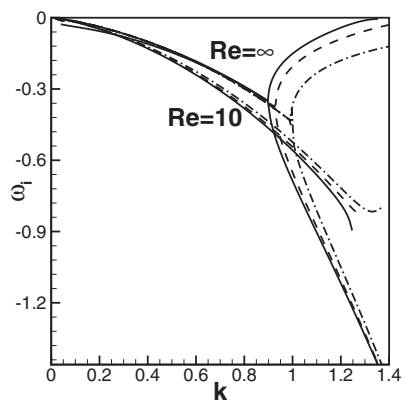

FIG. 16. Dispersion relations and decay rates for the gravitycapillary waves on the surface of a miscible interface for the fixed ratio $\mathrm{Ca} / \delta=1$. The results are shown for $\mathrm{Gr}=-1, \mathrm{Pe}=5$, for the inviscid and viscous $(\operatorname{Re}=10)$ cases, (a), (b) $A=-0.5$ and various $\mathrm{Ca}$ and $\delta(\mathrm{Ca}=\delta=0.02$, solid line; $\mathrm{Ca}=\delta=0.04$, dashed line; $\mathrm{Ca}=\delta=0.08$, dash-dot line); (c), (d) $A=0.5$ and various $\mathrm{Ca}$ and $\delta(\mathrm{Ca}=\delta=0.03$, solid line; $\mathrm{Ca}=\delta=0.06$, dashed line; $\mathrm{Ca}=\delta=0.12$, dash-dot line).

\section{CONCLUSIONS}

We have investigated the linear evolution of the small normal disturbances to a flat horizontal phase boundary separating two slowly miscible liquids. The phase-field approach was employed to model the thermo- and hydrodynamic evolution of the binary mixture. The Boussinesq approximation of the full Cahn-Hilliard-Navier-Stokes equations was used to define the amplitude equations for the small normal disturbances. We were interested in the binary mixtures with the upper critical point. The thermodynamic states of the binary mixture were defined by the Landau free energy function, with the main phenomenological parameter $A$, which is negative for the undercritical temperatures and positive for the temperatures above the critical point.

Two primary aims pursued by this work were to understand the limits of the phase-field approach and to understand the effects of interfacial diffusion on the dynamics of the 
Rayleigh-Taylor instability and on the dispersion and dissipation of the gravity-capillary waves.

The work was fulfilled in three main steps. First we considered the pure hydrodynamic evolution (under the condition that $\mathrm{Pe} \rightarrow \infty$ ) of a smeared interface. The results were validated through the use of the classical formulas obtained for sharp interfaces. We found that all classical expectations for the Rayleigh-Taylor instability and for the waves can be observed. In the case of the Rayleigh-Taylor instability (heavier liquid overlays a lighter one), all perturbations monotonically grow. The growth rates are reduced by the viscous and capillary forces. In the opposite configuration of a lighter liquid overlaying a heavier one, the gravity-capillary waves are developed, with the phase speed proportional to $k^{-1 / 2}$. The damping is defined by the viscous and capillary effects, reducing the range of the modes that exhibit the wavelike behavior.

The sharp-interface results can be reproduced for thinner interfaces, but the situation is however different for $\mathrm{Ca}=0$ and $\mathrm{Ca} \neq 0$. In the former case, the sharp interface results are achieved by a simple gradual reduction of the interface thickness. In the latter case, the interface thickness should be decreased simultaneously with the capillary number, so to keeping the ratio $\mathrm{Ca} / \delta$ (which is proportional to the surface tension coefficient) constant. Such procedures allow accurate reproduction of the eigenvalue spectrum of a sharp interface for the range of wave numbers limited by $k \delta \ll 1$. Thus, for instance, in order to reproduce the eigenvalue spectrum for $k \leqslant 5$, the interface thickness must be at least $\delta=0.001$. This condition can however be relaxed on the basis of the following reasons. To describe the development of the Rayleigh-Taylor instability we may need to define accurately only the fastest growing mode, so the condition becomes $k_{\max } \delta \ll 1$, or only the unstable modes, which are limited by the cutoff value $k_{c}=\sqrt{3 \delta \mathrm{Gr} / \mathrm{Ca}}$ due to the capillary action, and the condition becomes $k_{c} \delta \ll 1$. In the case of the gravity-capillary waves, we may either be interested in the wave of the particular wavelength, or in the modes that exhibit the wavelike behavior, which are limited by the bifurcation point.

Next, we considered the pure thermodynamic instability of a diffuse interface. Contrary to the research results currently available in the literature, we treated $\mathrm{Ca}$ and $\delta$ as two independent parameters (in other works the stability of the kink solution, with $\delta_{0}=\sqrt{-\mathrm{Ca} / A}$, i.e., existent for $A<0$, was investigated). We found that, for $A<0$, all thermodynamic perturbations monotonically decay for thin interfaces, when $\delta \leqslant \delta_{0}$, and, for $A>0$, all perturbations unconditionally decay. If however $A<0$ and $\delta>\delta_{0}$, the long-wave instability develops.

Finally, we considered the stability of miscible interfaces with respect to both thermo- and hydrodynamic perturbations. We found that the diffusivity introduces an additional dissipation, and its influence is very similar to the effects produced by the viscous force. The introduction of diffusivity slows the growth of the Rayleigh-Taylor instability; it also reduces the propagation speeds of the gravity-capillary waves and increases the damping of the waves.

In general, all three effects - the viscosity, diffusivity, and capillarity_damp perturbations, but these effects interplay and hence can emphasize or suppress each other. The cumulative viscous and diffusive damping is able to completely suppress the growth of the short-wavelength modes, which is not observed when these effects act separately. The strong viscous or diffusive damping can make the effect of capillarity practically nonexistent. That means that the use of the phasefield approach as a numerical method for describing the evolution of immiscible interfaces should in general take into account the correlations between the Reynolds, Peclet, and capillary numbers, and the interface thickness.

We also found a qualitative difference in the results obtained for $\mathrm{Ca}=0$ and $\mathrm{Ca} \neq 0$. For the case of negligible capillary effects, the evolution of small perturbations is totally determined by hydrodynamics. The diffusion is included by the diffusive flux defined by the Fickian law, but we found that the concentration field in this case mostly adjusts the variations of the velocity field. If however $\mathrm{Ca} \neq 0$, then the evolution of perturbations is dominated by the thermodynamic part of the mathematical problem. The difference in the results is even stronger for the gravity-capillary waves, when in the case of nonnegligible capillary effects and when $A<0$ and $\delta>\delta_{0}$, the thermodynamic long-wave instability makes the layer unstable even if the lighter liquid overlays the heavier one.

\section{ACKNOWLEDGMENTS}

This work was supported by EPSRC Projects No. EP/G014337 "Pore-level modeling of the miscible displacement" and No. EP/G069581/1 "UK Turbulence Consortium."
[1] S. J. W. L. Rayleigh, Proc. London Math. Soc. 14, 170 (1883).

[2] G. Taylor, Proc. R. Soc. London, Ser. A 201, 192 (1950).

[3] R. Bellman and R. Pennington, Quart. Appl. Math. 12, 151 (1954).

[4] C.-M. Tchen, J. Appl. Phys. 27, 760 (1956).

[5] W. H. Reid, Math. Proc. Cambridge Philos. Soc. 57, 415 (1961).

[6] S. Chandrasekhar, Hydrodynamic and Hydromagnetic Stability (Clarendon Press, Oxford, 1961).

[7] D. J. Lewis, Proc. R. Soc. London, Ser. A 202, 81 (1950).

[8] H. W. Emmons, C. T. Chang, and B. C. Watson, J. Fluid Mech. 7, 177 (1960).

[9] M. Ratafia, Phys. Fluids 16, 1207 (1973).
[10] F. Dias and C. Kharif, Annu. Rev. Fluid Mech. 31, 301 (1999).

[11] M. Perlin and W. W. Schultz, Annu. Rev. Fluid Mech. 32, 241 (2000).

[12] M. Mukhopadhyay, Natural Extracts Using Supercritical Carbon Dioxide (CRC Press LLC, Boca Raton, 2000).

[13] J. W. Jawitz, M. D. Annable, and P. S. C. Rao, J. Contam. Hydrol. 31, 211 (1998).

[14] A. Silva, C. Delerue-Matos, and A. Fiuza, J. Hazardous Mater. 124, 224 (2005).

[15] T. Babadagli, J. Petroleum Sci. Eng. 57, 221 (2007).

[16] M. S. P. Stevar and A. Vorobev, J. Colloid Interface Sci. 383, 184 (2012). 
[17] M. S. P. Stevar and A. Vorobev, Transp. Porous Media (unpublished).

[18] D. D. Joseph, Eur. J. Mech. B/Fluids 9, 565 (1990).

[19] S. E. May and J. V. Maher, Phys. Rev. Lett. 67, 2013 (1991).

[20] D. D. Joseph and Y. Y. Renardy, Fundamentals of Two-Fluid Dynamics. Part II: Lubricated Transport, Drops and Miscible Liquids (Springer-Verlag, Berlin, 1993).

[21] P. Petitjeans and P. Kurowski, C. R. Acad. Sci. Paris II B 325, 587 (1997).

[22] A. Pojman, C. Whitmore, M. L. T. Liveri, R. Lombardo, J. Marszalek, R. Parker, and B. Zoltowski, Langmuir 22, 2569 (2006).

[23] L. Lacaze, P. Guenoun, D. Beysens, M. Delsanti, P. Petitjeans, and P. Kurowski, Phys. Rev. E 82, 041606 (2010).

[24] J. Lowengrub and L. Truskinovsky, Proc. R. Soc. London, Ser. A 454, 2617 (1998).

[25] A. Vorobev, Phys. Rev. E 82, 056312 (2010).

[26] H. Hu and D. Joseph, Z. Angew. Math. Phys. 43, 626 (1992).

[27] S. H. Vanaparthy, E. Meiburg, and D. Wilhelm, J. Fluid Mech. 497, 99 (2003).

[28] Y. Gaponenko and V. Shevtsova, Acta Astronaut. 66, 174 (2010).

[29] L. D. Landau and E. M. Lifshitz, Course of Theoretical Physics, Volume 5, Statistical Physics (Elsevier, Butterworth-Heinemann, Oxford, 1980).

[30] C. W. Hirt and B. D. Nichols, J. Comput. Phys. 39, 201 (1981).

[31] S. Osher and J. A. Sethian, J. Comput. Phys. 79, 12 (1988).

[32] J. W. Cahn and J. E. Hilliard, J. Chem. Phys. 31, 688 (1959).

[33] D. Jasnow and J. Vinals, Phys. Fluids 8, 660 (1996).

[34] D. M. Anderson, G. B. McFadden, and A. A. Wheeler, Annu. Rev. Fluid Mech. 30, 139 (1998).

[35] D. Jacqmin, J. Comput. Phys. 155, 96 (1999).

[36] D. Jacqmin, J. Fluid Mech. 402, 57 (2000).

[37] H. Ding, P. D. M. Spelt, and C. Shu, J. Comput. Phys. 226, 2078 (2007).
[38] A. Celani, A. Mazzino, P. Muratore-Ginanneschi, and L. Vozzela, J. Fluid Mech. 622, 115 (2009).

[39] A. Shinozaki and Y. Oono, Phys. Rev. E 47, 804 (1993).

[40] D. Bettinson and G. Rowlands, Phys. Rev. E 54, 6102 (1996).

[41] A. Frischknecht, Phys. Rev. E 56, 6970 (1997).

[42] A. Riaz and E. Meiburg, Phys. Fluids 15, 938 (2003).

[43] L. D. Landau and E. M. Lifshitz, Course of Theoretical Physics, Volume 6, Fluid Mechanics (Elsevier, Butterworth-Heinemann, Oxford, 1987).

[44] J. D. V. der Waals, J. Stat. Phys. 20, 197 (1979).

[45] D. J. Korteweg, Archives Néerlandaises des Sciences Exactes et Naturelles 6, 1 (1901).

[46] J. W. Cahn and J. E. Hilliard, J. Chem. Phys. 28, 258 (1958).

[47] The parameter introduced here should not be mixed up with another classical parameter, defined as $\eta V / \sigma$, with $\eta, V$, and $\sigma$ being the viscosity, the typical fluid velocity, and the surface tension coefficient, respectively. This classical parameter is also called the capillary parameter and is used within the Laplacian models to define the ratio between the viscous and capillary forces. The introduced parameter will be also called the capillary parameter to underline that it is proportional to the capillary constant, and hence it defines the strength of the capillary effect. In some papers, e.g., [24], an alternative name, the Cahn number, is also used for this parameter.

[48] R. Bechov and A. Szewczyk, Phys. Fluids 6, 1391 (1963).

[49] D. S. Conte, SIAM Rev. 8, 309 (1966).

[50] L. M. Mack, J. Fluid Mech. 73, 497 (1976).

[51] A. Tumin, J. Fluids Eng. 125, 428 (2003).

[52] B. S. Ng and W. H. Reid, J. Comput. Phys. 58, 209 (1985).

[53] L. Allen and T. J. Bridges, Numer. Math. 92, 197 (2002).

[54] J. Humpherys, B. Standstede, and K. Zumbrun, Numer. Math. 103, 631 (2006). 